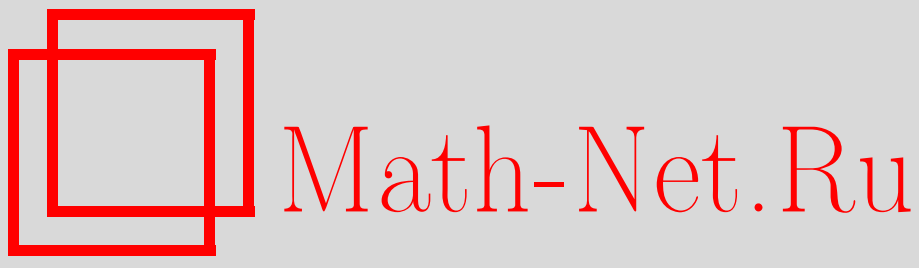

М. И. Анохин, О распознаваемости классов алгебраических систем за полиномиальное время, Матем. сб., 2002, том 193, номер 2, 3-34

DOI: https://doi.org/10.4213/sm625

Использование Общероссийского математического портала Math-Net.Ru подразумевает, что вы прочитали и согласны с пользовательским соглашением http://www . mathnet.ru/rus/agreement

Параметры загрузки:

IP: 52.6 .47 .48

26 апреля 2023 г., $11: 21: 04$ 


\author{
М.И. Анохин
}

\title{
О распознаваемости классов алгебраических систем за полиномиальное время
}

\begin{abstract}
Рассматриваются несколько видов распознаваемости классов алгебраических систем за полиномиальное время с использованием оракула, выполняющего сигнатурные операции и предикаты. Изучаются связи между этими видами распознаваемости. Доказано несколько результатов как о распознаваемости, так и о нераспознаваемости за полиномиальное время для некоторых конечно базируемых многообразий универсальных алгебр.

Библиографйл: 18 названий.
\end{abstract}

\section{§1. Введение}

1.1. Вычислительная модель. Пусть $A$ - непустая алгебраическая система фиксированной конечной сигнатуры $\Sigma$, носитель которой является подмножеством $\{0,1\}^{n}$ при некотором $n \geqslant 1$. Пусть также $\mathscr{A}$ - алгоритм, который может обрашаться с корректными запросами к оракулу, выполняющему сигнатурные операции и предикаты на $A$. На вход алгоритму $\mathscr{A}$ подается некоторая непустая последовательность $s=\left(s_{1}, \ldots, s_{m}\right)$ элементов $A$ (например, $s$ может быть произвольной непустой конечной системой порождаюших $A$ ). Алгоритмы такого вида мы называем О-алгоритмами (см. п. 3.1). Предполагается также, что алгоритм $\mathscr{A}$ полиномиален, т.е. время его работы на входе $s$ с использованием вьшеуказанного оракула не превосходит $c(m n)^{d}$ для некоторых констант $c>0$ и $d \geqslant 0$. В соответствии с классическим тезисом Эдмондса именно полиномиальные алгоритмы принято считать эффективными.

Такая вычислительная модель, по-видимому, была впервые использована для групा в работе Бабаи и Семереди [1] под названием модели групп типа "черного ящика" (black box group model). Аналогичная модель (для циклических групп) была введена Шупом в [2]; алгоритмы в этой модели он называет генерическими (generic). Из леммы 6.1 настояшей работы вытекает, что модель Шупа по сушеству эквивалентна рассматриваемой нами.

Используемый в настоящей работе подход к вычислительным задачам позволяет отвлечься от особенностей конкретных реализаций алгебраических систем, сосредоточив свое внимание на абстрактных алгебраических свойствах этих систем. Существуют и другие подходы, обладающие этим свойством (см., например, работы В.И. Нечаева [3] и Боне и Липтона [4]). Все эти подходы аналогичны обычному в алгебре подходу, при котором интересуются лишь алгебраической структурой рассматриваемой алгебраической системы (с точностью до изоморфизма), а не природой элементов ее носителя. 
1.2. Неформальная постановка задачи. В настоящей работе мы рассматриваем следующую задачу. Пусть $\mathfrak{C}$ и $\mathfrak{U}$ - некоторые фиксированные абстрактные классы алгебраических систем сигнатуры $\Sigma$. Предположим, что вышеуказанная алгебраическая система $A$ принадлежит $\mathfrak{U}$. Требуется определить (с помощью вышеуказанного алгоритма $\mathscr{A})$, принадлежит $A$ классу $\mathfrak{C}$ или не принадлежит. При этом нужно дать правильный ответ с вероятностью не менее $2 / 3$. Последняя константа может быть заменена на любое действительное число $q$, удовлетворяющее неравенствам $1 / 2<q<1$ (см. п. 3.2). Введем переменную $X$, которая может принимать произвольные значения из множества букв $\{\mathrm{G}, \mathrm{A}, \mathrm{R}\}$. В случае, когда $X=\mathrm{A}$, мы будем подразумевать (иногда не оговаривая этого особо), что все алгебраические системы из $\mathfrak{U}$ являются обобшенными группами (см. п. 2.8). Значение переменной $X$ определяет то, какие последовательности $s$ подаются на вход алгоритму $\mathscr{A}$. При $X=\mathrm{G}(X=\mathrm{A}) s$ есть произвольная непустая конечная система порождающих $A$ (соответственно аддитивной групшы $A$ ), а при $X=\mathrm{R}$ эта последовательность состоит из полиномиального от $n$ числа независимых случайных элементов относительно равномерного распределения на носителе $A$. Для групп случай $X=\mathrm{G}$ (равносильно, $X=\mathrm{A}$ ) рассматривался, в частности, в работах [1], [5] и [6], а случай $X=\mathrm{R}$ идейно аналогичен понятию полиномиально вычислимого семейства групп из работы Импальящо и Наора [7; определение 4.1]. Однако задачи, изучаемые в этих работах, большей частью отличны от вышеуказанной задачи. Отметим, что буквы $\mathrm{G}, \mathrm{A}$ и $\mathrm{R}$, являющиеся значениями переменной $X$, происходят от слов Generators, Additive generators и Random elements соответственно. Если существует решающий вышеуказанную задачу (детерминированньй) алгоритм $\mathscr{A}$, удовлетворяюший всем сделанньм выше предположениям, то мы называем класс $\mathfrak{C}$ ( детерминированно) $X$-распознаваемым в классе $\mathfrak{U}$.

Введем также переменную $Y$, которая может принимать произвольные значения из множества букв $\{\mathrm{G}, \mathrm{A}\}$. Как и для переменной $X$, условие $Y=$ А подразумевает (иногда без явного упоминания об этом), что все алгебраические системы из $\mathfrak{U}$ являются обобшенньми группами (см. п. 2.8). Мы определяем также сильную $Y$-распознаваемость (см. определение 3.3 ), из которой вытекает детерминированная $Y$-распознаваемость. Это вызвано тем, что многие из используемых в работе методов доказательства положительных результатов позволяют получить более сильные свойства, чем $Y$-распознаваемость. Термин "распознаваемость" будет обозначать как $X$-распознаваемость, так и детерминированную $X$-распознаваемость и сильную $Y$-распознаваемость. Для краткости мы говорим просто о распознаваемости, подразумевая под этим распознаваемость за полиномиальное время, если не оговорено противное.

Отметим, что в работе Бабаи и др. [5] доказано, что классы всех разрешимых и всех нильпотентных групп G-распознаваемы в классе всех групп. С другой стороны, из рассуждений Бабаи [6; 9 ] следует, что классы всех циклических и всех простых групп не являются G-распознаваемыми даже в классе элементарных абелевых $p$-групп, где простое число $p$ не фиксировано, а зависит от групшы. Здесь $\mathrm{G}$-распознаваемость можно заменить на $X$-распознаваемость при произвольном $X \in\{\mathrm{G}, \mathrm{A}, \mathrm{R}\}$ ввиду следствия 4.2. По мнению автора, особый интерес представляет вопрос о распознаваемости в классе $\mathfrak{U}$ для классов, которые являются конечно аксиоматизируемьми в $\mathfrak{U}$. Это объясняется тем, что такие классы в некотором 
смысле детерминированно G-распознаваемы и детерминированно R-распознаваемы в $\mathfrak{U}$ за экспоненциальное время (см. следствие 3.1). В настоящей работе мы в основном изучаем распознаваемость конечно базируемых многообразий универсальных алгебр.

1.3. Обзор работы. Параграф 2 содержит основные определения, обозначения и факты, используемые в работе. В $\S 3$ приведены точные описания вычислительной модели и постановки задачи (их краткое неформальное описание см. в пп. 1.1 и 1.2). Параграф 4 посвящен исследованию связей между различньми видами распознаваемости. В частности, показано, что если $\mathfrak{U}$ состоит из обобшенных групп, то A-распознаваемость $\mathfrak{C}$ в $\mathfrak{U}$ эквивалентна R-распознаваемости $\mathfrak{C}$ в $\mathfrak{U}$ (см. следствие 4.1). Известные автору связи между различными видами распознаваемости выражены диаграммой (13).

В $\S 5$ приведены результаты, утверждающие распознаваемость некоторых конечно базируемых многообразий универсальных алгебр. Пункты 5.1 и 5.2 содержат утверждения о распознаваемости, которые выводятся из известных результатов с помощью простых рассуждений. В п. 5.3 доказано, что любое конечно базируемое многообразие мультиколец с нильпотентньми аддитивными группами сильно А-распознаваемо в классе всех мультиколец (теорема 5.1). В частности, это дает сильную А-распознаваемость любого конечно базируемого многообразия линейных алгебр над произвольным ассоциативным коммутативным конечно порожденньм кольцом с единицей в классе всех таких алгебр (см. следствие 5.2). Доказательство вышеуказанного результата для многообразий мультиколец основано на применении к мультикольцевым тождествам известной в теории линейных алгебр процедуры линеаризации (см., например, [8; гл. 1, § 3, п. 3.1]) .

Рассмотрим теперь задачу построения примеров конечно базируемых многообразий, не являющихся распознаваемыми в классе $\mathfrak{U}$. В общем случае эта задачаможет быть решена легко. Например, нетрудно показать, что многообразия коммутативных группоидов и полугрупп не являются ни G-распознаваемыми, ни R-pacпознаваемыми в классе всех группоидов. Легко также видеть, что многообразия всех полугрупп левых и правых нулей (т.е. многообразия полугруп, определенные тождествами $t_{1} t_{2}=t_{1}$ и $t_{1} t_{2}=t_{2}$ соответственно) сильно G-распознаваемы, но не R-распознаваемы в классе всех полугрупп. В то же время если $\mathfrak{U}-$ класс всех групп или класс всех линейных алгебр над каким-либо ассоциативным коммутативным конечно порожденным кольцом с единицей, то автору неизвестны примеры конечно базируемых подмногообразий $\mathfrak{U}$, не являющихся G-распознаваемыми в этом классе. Используя метод из $\S 9$ работы [6], можно показать, что квазимногообразие групп, определенное в многообразии $\mathfrak{N}_{2}$ всех нильпотентных групп класса не более 2 квазитождеством

$$
\left[t_{1}, t_{2}\right]=\left[t_{3}, t_{4}\right] \&\left[t_{1}, t_{3}\right]=1 \&\left[t_{2}, t_{3}\right]=1 \Longrightarrow\left[t_{1}, t_{2}\right]=1
$$

(где $\left.[x, y]=x^{-1} y^{-1} x y\right)$, не является $X$-распознаваемым в $\mathfrak{N}_{2}$ для всех $X \in$ $\{\mathrm{G}, \mathrm{A}, \mathrm{R}\}$ (здесь, как и выше, $X$ - распознаваемость не зависит от значения $X$ ввиду следствия 4.2).

Пусть $\mathfrak{V}$ - многообразие, определенное либо тождеством $t_{1}^{e}=0$ в многообразии всех полугрупп с нулем, либо одним из тождеств $t_{1}^{e} t_{2}=t_{1}^{e}$ или $t_{2} t_{1}^{e}=t_{1}^{e}$ в 
многообразии всех полугрупп, где $e$ - произвольное целое число, не меньшее 2. Параграф 7 посвящен доказательству того, что $\mathfrak{V}$ не является ни G-распознаваемњм, ни R-распознаваемым в некотором классе полугрупп с нулем. При этом используются понятия и результаты из вспомогательного $\S 6$.

\section{§2. Определения, обозначения и необходимые факты}

2.1. Множества и функции. Мы будем пользоваться стандартными обозначениями $\mathbb{Z}$ и $\mathbb{R}$ для множеств всех целых и всех действительных чисел соответственно. Пусть также $\mathbb{N}_{k}=\{n \in \mathbb{Z}: n \geqslant k\}(k \in \mathbb{Z}), \mathbb{I}_{m}=\{1, \ldots, m\}$ $\left(m \in \mathbb{N}_{0}\right), \mathbb{B}=\{0,1\}$ и $\mathbb{R}_{+}=\{x \in \mathbb{R}: x>0\}$. Обозначение log всегда подразумевает логарифм по основанию 2 . Как обычно, $\lceil x\rceil=\min \{n \in \mathbb{Z}: n \geqslant x\}$ и $\lfloor x\rfloor=\max \{n \in \mathbb{Z}: n \leqslant x\}$ для произвольного $x \in \mathbb{R}$. Функцию из $\mathbb{N}_{1}$ в $\mathbb{R}_{+}$вида $n \mapsto c n^{d}$, где $c \in \mathbb{R}_{+}$и $d \in \mathbb{R}_{+} \cup\{0\}$, мы называем полиномиальной. Если $c \in \mathbb{N}_{1}$ и $d \in \mathbb{N}_{0}$, то полиномиальная функция $n \mapsto c n^{d}$ назьвается целочисленной. Мы также будем называть әкспоненциальной функцию из $\mathbb{N}_{1}$ в $\mathbb{R}_{+}$вида $n \mapsto c d^{n}$, где $c, d \in \mathbb{R}_{+}$и $d>1$.

В настоящей работе буква $e$ не обозначает основание натуральных логарифмов; результат возведения этого основания в $x$-ю степень будет записываться в виде $\exp x$.

2.2. Последовательности. Пусть $A$ - некоторое множество и $n \in \mathbb{N}_{0}$. Через $A^{n}$ мы будем обозначать $n$-ю декартову степень множества $A$, т.е. множество всех последовательностей вида $\left(a_{1}, \ldots, a_{n}\right)$, где $a_{i} \in A$ для всех $i \in \mathbb{I}_{n}$. В частности, $A^{0}$ есть одноэлементное множество, состоящее из пустой последовательности. Положим $A^{*}=\bigcup_{n=0}^{\infty} A^{n}, A^{+}=\bigcup_{n=1}^{\infty} A^{n}$ и $A^{\geqslant m}=\bigcup_{n=m}^{\infty} A^{n}$, где $m \in \mathbb{N}_{0}$. Пусть также $|s|$ и $E(s)$ обозначают соответственно длину и множество всех элементов последовательности $s \in A^{*}$. Другими словами, если $s=\left(s_{1}, \ldots, s_{n}\right) \in A^{*}$, то $|s|=n$ и $E(s)=\left\{s_{1}, \ldots, s_{n}\right\}$. Отметим, что вертикальньми чертами будут обозначаться также мощность множества и модуль действительного числа, но значение этих символов всегда будет ясно из контекста.

$\mathrm{C}$ каждьм отображением $\varphi: A \rightarrow B$ можно естественньм образом связать отображение из $A^{*}$ в $B^{*}$, переводящее $\left(a_{1}, \ldots, a_{n}\right)$ в $\left(\varphi\left(a_{1}\right), \ldots, \varphi\left(a_{n}\right)\right)$, где $a_{i} \in A$ для всех $i \in \mathbb{I}_{n}\left(n \in \mathbb{N}_{0}\right)$. Это отображение мы будем обозначать так же, как и исходное.

2.3. Случайные величины. В настоящей работе мы будем иметь дело только с дискретными случайными величинами, т.е. принимающими значения на конечных или счетных множествах. Случайные величины, как правило, будут обозначаться символами с тильдой. Если не оговорено противное, то вводимые нами случайные величины предполагаются независимыми от всех других.

Пусть $\widetilde{a}$ - случайная величина, принимающая значения на некотором (конечном или счетном) множестве $A$. Тогда для $n \in \mathbb{N}_{0}$ через $\widetilde{a}^{(n)}$ будет обозначаться случайная величина $\left(\widetilde{a}_{1}, \ldots, \widetilde{a}_{n}\right)$ (со значениями в множестве $\left.A^{n}\right)$, где случайные величины $\widetilde{a}_{1}, \ldots, \widetilde{a}_{n}$ независимы и имеют то же самое распределение, что и $\widetilde{a}$. Запись вида $\widetilde{b} \in \mathrm{U} B$ означает, что $\widetilde{b}$ - случайная величина, распределенная равномерно на непустом конечном множестве $B$. 
Для произвольных случайных величин $\widetilde{u}$ и $\widetilde{v}$, принимающих значения на одном и том же конечном или счетном множестве $A$, положим

$$
\operatorname{dist}(\widetilde{u}, \widetilde{v})=\frac{1}{2} \sum_{a \in A}|\mathrm{P}(\widetilde{u}=a)-\mathrm{P}(\widetilde{v}=a)|=\max _{M \subseteq A}|\mathrm{P}(\widetilde{u} \in M)-\mathrm{P}(\widetilde{v} \in M)|
$$

(см. также, например, [9; определение 13.6]). Приведем некоторые свойства функции dist.

ЛЕмма 2.1. Пусть $\widetilde{u}, \widetilde{v}$ и $\widetilde{w}-$ случайные величины со значениями в некотором конечном или счетном множестве $A$, a $\widetilde{r}$ - случайная величина со значениями в некотором конечном или счетном множестве $B$. Тогда

1) $\operatorname{dist}(\widetilde{u}, \widetilde{w}) \leqslant \operatorname{dist}(\widetilde{u}, \widetilde{v})+\operatorname{dist}(\widetilde{v}, \widetilde{w})$

$2) \operatorname{dist}((\widetilde{u}, \widetilde{r}),(\widetilde{v}, \widetilde{r}))=\operatorname{dist}((\widetilde{r}, \widetilde{u}),(\widetilde{r}, \widetilde{v}))=\operatorname{dist}(\widetilde{u}, \widetilde{v})$, если $\widetilde{u}$ и $\widetilde{r}$ независимь и $\widetilde{v}$ и $\widetilde{r}$ независимы ;

3) $\operatorname{dist}(\varphi(\widetilde{u}), \varphi(\widetilde{v})) \leqslant \operatorname{dist}(\widetilde{u}, \widetilde{v})$ для любой функиии $\varphi: A \rightarrow B$;

4) если $S$ - событие в вероятностном пространстве, на котором определена случайная величина $(\widetilde{u}, \widetilde{v})$, такое, что $\mathrm{P}(\widetilde{u}=a, S)=\mathrm{P}(\widetilde{v}=a, S)$ для всех $a \in A$, то $\operatorname{dist}(\widetilde{u}, \widetilde{v}) \leqslant \mathrm{P}\left(S^{\prime}\right)$, где $S^{\prime}-$ дополнение $S$ в вышеуказанном вероятностном пространстве;

$5) \operatorname{dist}\left(\widetilde{u}^{(n)}, \widetilde{v}^{(n)}\right) \leqslant n \operatorname{dist}(\widetilde{u}, \widetilde{v})$ для любого $n \in \mathbb{N}_{0}$.

Пункты 1)-4) леммы 2.1 проверяются непосредственно, а п. 5) легко доказывается индукцией по $n$ с использованием пп. 1) и 2).

2.4. Алгебраические системы. Фиксируем произвольную сигнатуру $\Sigma$, которая может содержать символы как операций (в том числе 0-арных, которые играют роль констант), так и предикатов. Если не оговорено противное, то рассматриваемые алгебраические системы есть системы сигнатуры $\Sigma$. Кроме того, все алгебраические системы в настоящей работе предполагаются непустыми. Пусть также $\Omega(\Pi)$ - множество всех символов операций (соответственно предикатов) из $\Sigma$. Через $\operatorname{ar} \sigma$, где $\sigma \in \Sigma$, мы будем обозначать арность операции или предиката, соответствуюшего символу $\sigma$.

Следуя традиции, сложившейся в алгебре, алгебраические системы и их носители мы обозначаем одинаково, если это не вызывает недоразумений. Через Gen $A$, где $A$ - произвольная алгебраическая система, будет обозначаться множество всех последовательностей из $A^{+}$, порождающих систему $A$.

Предположим, что $\varphi$ - некоторое инъективное отображение алгебраической системы $A$ на множество $\varphi(A)$. Тогда $\varphi(A)$ может быть единственньм образом превращено в алгебраическую систему так, чтобы $\varphi$ стало изоморфизмом. В этом случае $\varphi(A)$ будет обозначать также эту алгебраическую систему.

Пусть $\mathfrak{C}$ и $\mathfrak{U}$ - некоторые классы алгебраических систем. Все классы алгебраических систем в настоящей работе предполагаются абстрактньми (т.е. содержащими вместе с каждой алгебраической системой все изоморфные ей системы) и состоящими из систем одной и той же сигнатуры $\Sigma$, если не оговорено противное. Для произвольной алгебраической системы $A$ положим

$$
\chi(A, \mathfrak{C})=\left\{\begin{array}{l}
1, \text { если } A \in \mathfrak{C} ; \\
0, \text { если } A \notin \mathfrak{C} .
\end{array}\right.
$$


Напомним, что класс алгебраических систем $\mathfrak{C}$ называется конечно аксиоматизируемым в классе алгебраических систем $\mathfrak{U}$, если $\mathfrak{C}$ может быть задан в $\mathfrak{U}$ конечным числом замкнутых формул первого порядка сигнатуры $\Sigma$. Многообразие, которое может быть задано в многообразии $\mathfrak{U}$ конечным числом тождеств, называется конечно базируемым в $\mathfrak{U}$. Конечная базируемость без указания многообразия, в котором она имеет место, означает конечную базируемость в классе всех алгебраических систем (сигнатуры $\Sigma$ ). Как правило, мы рассматриваем подмногообразия некоторого конечно базируемого многообразия $\mathfrak{U}$ (например, многообразия всех групп, всех полугрупп и т.п.). Очевидно, что для таких многообразий конечная базируемость (в классе всех алгебраических систем) равносильна конечной базируемости в $\mathfrak{U}$.

Как обычно, мы считаем, что сигнатура групп состоит из символов умножения, обращения и единицы. Пусть $K$ - произвольное ассоциативное коммутативное конечно порожденное кольцо с единицей. Тогда сигнатура линейных алгебр над $K$ предполагается состоящей из символов сложения, аддитивного обращения, нуля, умножения и действия элементов из некоторой конечной системы порождающих кольца $K$. Кроме того, последняя сигнатура может также содержать символ единицы. Таким образом, сигнатура линейных алгебр над $K$ конечна. Кроме того, легко показать (используя теорему Гильберта о базисе), что многообразие всех линейных алгебр над $K$ конечно базируемо (относительно вьшшеуказанной сигнатуры). Кольца мы рассматриваем как линейные алгебры над кольцом целых чисел.

2.5. Термы. Фиксируем счетное множество $T=\left\{t_{1}, t_{2}, \ldots\right\}$ предметных переменных. Обозначим через $\operatorname{Tm}_{\infty}(\Omega)$ множество всех термов сигнатуры $\Omega$ от переменных из $T$. Пусть также $\operatorname{Tm}_{m}(\Omega)$ - множество всех термов из $\operatorname{Tm}_{\infty}(\Omega)$, содержащих лишш переменные из $\left\{t_{1}, \ldots, t_{m}\right\}\left(m \in \mathbb{N}_{0}\right)$.

$\mathrm{C}$ каждым термом $v \in \operatorname{Tm}_{\infty}(\Omega)$ мы будем связывать его степень $\operatorname{deg} v$, равную числу вхождений в $v$ переменных из $T$, и множество nvar $v$ номеров переменных, входящих в $v$. Эти параметры определяются индукцией по построению терма следуюшим образом:

$$
\begin{aligned}
\operatorname{deg} t_{n} & =1, \quad \operatorname{deg} \omega\left(v_{1}, \ldots, v_{\operatorname{ar} \omega}\right)=\sum_{i=1}^{\operatorname{ar} \omega} \operatorname{deg} v_{i} \\
\operatorname{nvar} t_{n}=\{n\}, \quad \operatorname{nvar} \omega\left(v_{1}, \ldots, v_{\operatorname{ar} \omega}\right) & =\bigcup_{i=1}^{\operatorname{ar} \omega} \operatorname{nvar} v_{i},
\end{aligned}
$$

где $n \in \mathbb{N}_{1}, \omega \in \Omega, v_{1}, \ldots, v_{\text {ar } \omega} \in \operatorname{Tm}_{\infty}(\Omega)$. Индукцией по построению терма $v$ легко показать, что

$$
|\operatorname{nvar} v| \leqslant \operatorname{deg} v
$$

для любого $v \in \operatorname{Tm}_{\infty}(\Omega)$.

2.6. Группы. Пусть $G$ - произвольная группа. Для $a, b, a_{1}, \ldots, a_{n} \in G$ мы полагаем $[a, b]=a^{-1} b^{-1} a b$ (в аддитивной записи $\left.[a, b]=-a-b+a+b\right)$ и

$$
\left[a_{1}, \ldots, a_{n}\right]=\left[\ldots\left[\left[a_{1}, a_{2}\right], a_{3}\right], \ldots, a_{n}\right]
$$


Если $n \in \mathbb{N}_{1}$, то $G_{(n)}$ обозначает $n$-й член нижнего центрального ряда группы $G$, т.е. подгруппу, порожденную системой

$$
\left\{\left[a_{1}, \ldots, a_{n}\right]: a_{i} \in G, i \in \mathbb{I}_{n}\right\} .
$$

2.7. Дистрибутивность. Пусть $A$ и $B$ - некоторые группоиды, а $\varphi$ - отображение из $A^{n}$ в $B$, где $n \in \mathbb{N}_{0}$. Отображение $\varphi$ называется дистрибутивныцм на $A$, если

$$
\begin{aligned}
& \varphi\left(a_{1}, \ldots, a_{i-1}, a_{i} a_{i}^{\prime}, a_{i+1}, \ldots, a_{n}\right) \\
& \quad=\varphi\left(a_{1}, \ldots, a_{i-1}, a_{i}, a_{i+1}, \ldots, a_{n}\right) \varphi\left(a_{1}, \ldots, a_{i-1}, a_{i}^{\prime}, a_{i+1}, \ldots, a_{n}\right)
\end{aligned}
$$

для любого $i \in \mathbb{I}_{n}$ и любых $a_{1}, \ldots, a_{i-1}, a_{i}, a_{i}^{\prime}, a_{i+1}, \ldots, a_{n} \in A$.

Сформулируем следующее хорошо известное в теории групп утверждение, которое может быть легко доказано индукцией по $n$ с использованием коммутаторного тождества 33.34 (1) из [10].

Лемма 2.2. Пусть $G$ - произвольная группа и $n \in \mathbb{N}_{1}$. Тогда отображсение

$$
\left(a_{1}, \ldots, a_{n}\right) \mapsto\left[a_{1}, \ldots, a_{n}\right] G_{(n+1)} \quad\left(a_{i} \in G, \quad i \in \mathbb{I}_{n}\right)
$$

из $G^{n}$ в $G_{(n)} / G_{(n+1)}$ дистрибутивно на $G$.

2.8. Обобщенные группы и мультикольца. Предположим, что фиксированы символы $\mu, \iota, \zeta \in \Omega$ такие, что $\operatorname{ar} \mu=2, \operatorname{ar} \iota=1$ и $\operatorname{ar} \zeta=0$. Пусть также $\Theta=\Omega \backslash\{\mu, \iota, \zeta\}$. Назовем алгебраическую систему $A$ обобщенной группой, если $(A ; \mu, \iota, \zeta)$ является группой.

Пусть $A$ - обобшенная группа. Тогда группу $(A ; \mu, \iota, \zeta)$ мы будем называть аддитивной группой обобщенной группы $A$ и обозначать через $\operatorname{Add} A$. Если $\Sigma \neq\{\mu, \iota, \zeta\}$, то для этой группы будет использоваться аддитивная запись, т.е. вместо $\mu\left(a, a^{\prime}\right), \iota(a)$ и $\zeta$ мы будем писать $a+a^{\prime},-a$ и 0 соответственно. В противном случае речь идет об обычных группах, для которых будет использоваться (и уже использовалась) мультипликативная запись $\left(a a^{\prime}, a^{-1}\right.$ и 1 вместо $\mu\left(a, a^{\prime}\right)$, $\iota(a)$ и $\zeta$ соответственно). Отметим, что группа $A d d d$ не предполагается абелевой, несмотря на свое название.

Подмножество $I$ обобшенной группы $A$ называется идеалом в $A$, если $I-$ нормальная подгруппа группы $\operatorname{Add} A$ и отношение смежности по $I$ является конгруэнцией на $(A ; \Theta)$. Легко видеть, что конгруэнции на обобщенной группе $A$ - это отношения смежности (в группе $\operatorname{Add} A$ ) по всевозможным идеалам в $A$ и только они. Поэтому имеет смысл понятие фактора обобщенной группы $A$ по идеалу $I$; как обычно, этот фактор обозначается через $A / I$.

Для множества $S \subseteq A$ через $\left\langle\langle S\rangle_{A}\right.$ мы будем обозначать идеал в обобщенной группе $A$, порожденный множеством $S$. Напомним, что класс универсальных алгебр называется предмногообразием (или реплично полным классом), если он замкнут относительно взятия подалгебр и декартовых произведений и содержит одноэлементную алгебру. Если $\Pi=\varnothing, \mathfrak{P}$ - предмногообразие обобщенных групп и

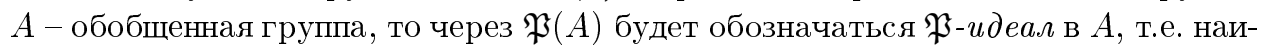
меньший элемент множества всех идеалов $I$ в $A$ таких, что $A / I \in \mathfrak{P}$. 
Рассмотрим обобщенные группы в случае, когда $\Pi=\varnothing$. Тогда обобщенная группа $A$ назьвается мультикольиом, если операция на $A$, соответствующая произвольному символу $\theta \in \Theta$ такому, что $\operatorname{ar} \theta \geqslant 1$, дистрибутивна на $(A ; \mu)$. Это определение совпадает с определением мультиоператорного кольца из [11; гл. II, $\S 1]$, а термин "мультикольцо" взят из [12; гл. $1, \S 2$, п. 2.1]. Понятие мультикольца (мультиоператорного кольца) достаточно известно в алгебре (оно является обобщением понятий группы, модуля, кольца и алгебры). Однако определение этого понятия, как правило, предполагает, что $\Theta$ не содержит символов 0-арных операций (см., например, $[12 ;$ гл. $1, \S 2$, п. 2.1]). Мы допускаем случай, когда $\Theta$ содержит такие символы, но не требуем дистрибутивности от операций, соответствующих этим символам (очевидно, что 0-арная операция, дистрибутивная на произвольной группе, равна единице этой группы).

\section{§3. Постановка задачи}

В этом параграфе мы будем предполагать, что сигнатура $\Sigma$ конечна. Это предположение позволяет избежать проблем, связанных с использованием элементов $\Sigma$ алгоритмами.

Все алгоритмы в настоящей работе будут обозначаться рукописными буквами. Рассматриваемые алгоритмы, вообще говоря, являются вероятностньми. Это значит, что они могут обращаться к оракулу RB, дающему независимые случайные (относительно равномерного распределения на $\mathbb{B}$ ) биты. Отметим, что детерминированные алгоритмы (не использующие этот оракул) мы также относим к числу вероятностных.

Функция $\varphi$ на множестве $\mathbb{N}_{1}$ называется полиномиально вычислимой, если существуют полиномиальная функция $\rho$ и детерминированный алгоритм (не использующий никаких оракулов), который на произвольном входе $n \in \mathbb{N}_{1}$ вычисляет значение $\varphi(n)$ за время, не превосходящее $\rho(n)$.

3.1. О-алгоритмы. Для алгебраической системы $A \subseteq \mathbb{B}^{n}\left(n \in \mathbb{N}_{1}\right)$ обозначим через $O_{A}$ оракул, который на запрос $(\sigma, a)$, где $\sigma \in \Sigma$ и $a \in A^{\text {ar } \sigma}$, возврашает значение $\sigma(a)$ в $A$. Запрос вышеуказанного вида мы будем называть корректным. Назовем алгоритм О-алгоритмом, если при вычислении на произвольном входе $s \in A^{+}$, где $A$ - произвольная алгебраическая система вышеуказанного вида, он имеет доступ к оракулу $O_{A}$ и делает только корректные запросы к этому оракулу.

Пусть $\mathscr{A}$ - произвольный О-алгоритм и $n \in \mathbb{N}_{1}$. Введем следующие обозначения:

1) $\operatorname{RT}(\mathscr{A}, n)$ - супремум времени работы $\mathscr{A}$ на входах $s \in A^{+}\left(A \subseteq \mathbb{B}^{n^{\prime}}\right.$ алгебраическая система, $\left.n^{\prime} \in \mathbb{N}_{1},|s| n^{\prime}=n\right)$ с использованием оракула $O_{A}$;

2) $\operatorname{NRB}(\mathscr{A}, n)$ - супремум числа запросов $\mathscr{A}$ к оракулу RB при вычислениях на входах $s \in A^{+}\left(A \subseteq \mathbb{B}^{n^{\prime}}\right.$ - алгебраическая система, $\left.n^{\prime} \in \mathbb{N}_{1},|s| n^{\prime}=n\right)$ с использованием оракула $O_{A}$;

3) $\operatorname{NOQ}(\mathscr{A}, n)$ - супремум числа запросов $\mathscr{A}$ к оракулу $O_{A}$ при вычислениях на входах $s \in A^{+}\left(A \subseteq \mathbb{B}^{n^{\prime}}\right.$ - алгебраическая система, $\left.n^{\prime} \in \mathbb{N}_{1},|s| n^{\prime}=n\right)$ с использованием оракула $O_{A}$.

Супремумы здесь берутся по всевозможным алгебраическим системам $A \subseteq \mathbb{B}^{n^{\prime}}$, последовательностям $s \in A^{+}$таким, что $|s| n^{\prime}=n$, и ответам оракула RВ на за- 
просы О-алгоритма $\mathscr{A}$. В работе рассматриваются только такие О-алгоритмы $\mathscr{A}$, что для всех $n \in \mathbb{N}_{1}$ значение $\operatorname{RT}(\mathscr{A}, n)$ конечно. Следовательно, конечными являются значения $\operatorname{NRB}(\mathscr{A}, n)$ и $\operatorname{NOQ}(\mathscr{A}, n)$, так как

$$
\operatorname{NRB}(\mathscr{A}, n), \operatorname{NOQ}(\mathscr{A}, n) \leqslant \operatorname{RT}(\mathscr{A}, n)
$$

для любого $n \in \mathbb{N}_{1}$.

О-алгоритм $\mathscr{A}$ называется полиномиальным (әкспоненциальным ), если сушествует полиномиальная функция $\rho$ такая, что $\operatorname{RT}(\mathscr{A}, n) \leqslant \rho(n)$ (соответственно $\operatorname{RT}(\mathscr{A}, n) \leqslant \exp (\rho(n)))$ для всех $n \in \mathbb{N}_{1}$.

ЗАмечание 3.1. Пусть $A \subseteq \mathbb{B}^{n}\left(n \in \mathbb{N}_{1}\right)$ - алгебраическая система. В определении полиномиального О-алгоритма требуется, чтобы время его работы на входе $s$ не превосходило значения $\rho(|s| n)$ при некоторой полиномиальной функции $\rho$ для произвольной последовательности $s \in A^{+}$, где алгебраическая система $A$ вышеуказанного вида также произвольна. В то же время для рассматриваемых нами задач сушественны лишш вычисления О-алгоритмов на входах из некоторых множеств $I(A) \subseteq A^{+}$при $A \in \mathfrak{U}$, где $\mathfrak{U}$ - некоторый класс алгебраических систем. Однако для любого О-алгоритма $\mathscr{A}$, время работы которого не превосходит $\rho(|s| n)$ (где $\rho$ - полиномиальная функция) лишш на входах $s \in I(A)$ при $A \in \mathfrak{U}$, существует полиномиальный (в вышеуказанном смысле) О-алгоритм $\mathscr{B}$, работаюший на входах из $I(A)$ при $A \in \mathfrak{U}$ так же, как и $\mathscr{A}$. Этот О-алгоритм $\mathscr{B}$ строится следуюшим образом. Не ограничивая обшности, мы считаем, что полиномиальная функция $\rho$ целочисленна. Тогда О-алгоритм $\mathscr{B}$ на произвольном входе $s \in A^{+}(A-$ произвольная алгебраическая система вышеуказанного вида) выполняет О-алгоритм $\mathscr{A}$ на этом входе, считая число шагов, сделанных $\mathscr{A}$. Если $\mathscr{A}$ закончил вычисление не более чем за $\rho(|s| n)$ шагов, то $\mathscr{B}$ также заканчивает вычисление и выдает выходное значение О-алгоритма $\mathscr{A}$. В противном случае О-алгоритм $\mathscr{B}$ прекрашает вычисление и выдает произвольное выходное значение. Аналогичные соображения справедливы также для экспоненциальных О-алгоритмов.

Имея в виду замечание 3.1 , при построении полиномиальных и экспоненциальных О-алгоритмов мы будем описывать их работу только на тех входах, которые существенны для данной задачи. Время работы этих О-алгоритмов будет очевидньм образом оцениваться сверху значением функции соответствующего вида на $|s| n$.

Пусть $A \subseteq \mathbb{B}^{n}\left(n \in \mathbb{N}_{1}\right)$ - алгебраическая система и $s \in A^{+}$. Через $\mathscr{A}(A, s)$ будет обозначаться выходное значение О-алгоритма $\mathscr{A}$ при вычислении на входе $s$ с использованием оракула $O_{A}$ (и оракула $\mathrm{RB}$, разумеется). Это выходное значение является случайной величиной, зависящей от ответов оракула $\mathrm{RB}$ на запро-

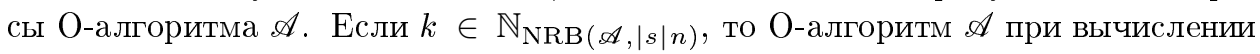
на входе $s$ в качестве источника случайных битов вместо оракула RB может использовать значение случайной последовательности $\widetilde{r} \in \mathrm{U} \mathbb{B}^{k}$, подаваемое этому О-алгоритму в качестве дополнительного входа. Можно рассматривать вычисление О-алгоритма $\mathscr{A}$ на входе $s$, когда в качестве вышеуказанного дополнительного входа этому О-алгоритму подается произвольная последовательность из $\mathbb{B} \geqslant \operatorname{NRB}(\mathscr{A},|s| n)$. Выходное значение О-алгоритма $\mathscr{A}$ при вычислении на входе $s$ с использованием оракула $O_{A}$ и последовательности битов $r \in \mathbb{B} \geqslant \operatorname{NRB}(\mathscr{A},|s| n)$ как 
дополнительного входа мы будем обозначать через $\mathscr{A}(A, s, r)$. Это значение зависит от $A, s$ и $r$ детерминированно.

Фиксируем некоторые классы алгебраических систем $\mathfrak{C}$ и $\mathfrak{U}$. Напомним, что переменные $X$ и $Y$ могут принимать произвольные значения из множеств букв $\{\mathrm{G}, \mathrm{A}, \mathrm{R}\}$ и $\{\mathrm{G}, \mathrm{A}\}$ соответственно. Если $X=\mathrm{A}$ или $Y=\mathrm{A}$, то предполагается (иногда без явного упоминания об этом), что все алгебраические системы из $\mathfrak{U}$ являются обобшенньми группами. Для единообразия назовем $Y$-порожсдающей последовательностью алгебраической системы $A \in \mathfrak{U}$ произвольную последовательность из Gen $A$, если $Y=\mathrm{G}$, и произвольную последовательность из Gen Add $A$, если $Y=$ А. Другими словами, конечная последовательность элементов алгебраической системы $A \in \mathfrak{U}$ является $\mathrm{G}$-порождающей (А-порождающей) последовательностью этой системы, если и только если она непуста и порождает систему $A$ (соответственно аддитивную группу обобшенной группы $A$ ).

\section{2. $X$-распознаваемость.}

ОПРЕДЕЛЕНИЕ 3.1. Класс $\mathfrak{C}$ называется (детерминированно) Y-распознаваeмым в классе $\mathfrak{U}$, если существует полиномиальньй (детерминированньй) О-алгоритм $\mathscr{A}$ такой, что

$$
\mathrm{P}(\mathscr{A}(A, s)=\chi(A, \mathfrak{C})) \geqslant \frac{2}{3}
$$

для любой алгебраической системы $A \subseteq \mathbb{B}^{n}$ из $\mathfrak{U}\left(n \in \mathbb{N}_{1}\right)$ и любой $Y$-порождаюшей последовательности $s$ системы $A$.

Смысл равенства $\mathscr{A}(A, s)=\chi(A, \mathfrak{C})$ в определении 3.1 (и аналогичных равенств ниже) состоит в том, что О-алгоритм $\mathscr{A}$ при вычислении на входе $s$ с использованием оракула $O_{A}$ (и, вообше говоря, оракула $\mathrm{RB}$ ) выдал правильный ответ на вопрос о том, принадлежит алгебраическая система $A$ классу $\mathfrak{C}$ или нет.

ЗАмечАниЕ 3.2. Очевидно, что класс $\mathfrak{C}$ детерминированно $Y$-распознаваем в классе $\mathfrak{U}$, если и только если существует полиномиальный детерминированньй О-алгоритм $\mathscr{A}$ такой, что $\mathscr{A}(A, s)=\chi(A, \mathfrak{C})$ для любой алгебраической системы $A \subseteq \mathbb{B}^{n}$ из $\mathfrak{U}\left(n \in \mathbb{N}_{1}\right)$ и любой $Y$-порождающей последовательности $s$ системы $A$.

ОПРЕДЕЛЕНИЕ 3.2. Класс $\mathfrak{C}$ называется (детерминированно) R-распознаваeмым в классе $\mathfrak{U}$, если существуют полиномиальньй (детерминированный) О-алгоритм $\mathscr{A}$ и целочисленная полиномиальная функция $\rho$ такие, что для любой алгебраической системы $A \subseteq \mathbb{B}^{n}$ из $\mathfrak{U}\left(n \in \mathbb{N}_{1}\right)$ справедливо неравенство

$$
\mathrm{P}(\mathscr{A}(A, \widetilde{a})=\chi(A, \mathfrak{C})) \geqslant \frac{2}{3}
$$

в котором $\widetilde{a} \in \mathrm{U} A^{\rho(n)}$.

ЗАмЕчАнИЕ 3.3. Можно считать, что алгоритм из определения 3.2 в качестве источника случайных элементов алгебраической системы $A \subseteq \mathbb{B}^{n}$ из $\mathfrak{U}\left(n \in \mathbb{N}_{1}\right)$ использует не значение входной последовательности $\widetilde{a} \in_{\mathrm{U}} A^{\rho(n)}$, а дополнительньй оракул. Эти два подхода аналогичны двум подходам к обеспечению О-алгоритмов случайными битами (см. п. 3.1). 
Определения $Y$-распознаваемости и (детерминированной) R-распознаваемости напоминают определение сложностного класса ВPР (см., например, [9; определение 7.5], [13; определение 3.1]). Для этих видов распознаваемости можно дать следуюшие эквивалентные определения, аналогичные приведенньм в утверждениях 7.3.1 и 7.3.2 из [9] для класса ВРP.

ПРЕДЛОЖЕНИЕ 3.1. Класс $\mathfrak{C}$ является $Y$-распознаваемым в классе $\mathfrak{U}$ тогда и только тогда, когда выполнено любое из следующих әквивалентных условий:

1) существуют полиномиальный О-алгоритм $\mathscr{A}$, полиномиальный детерминированный О-алгоритм $\mathscr{B}$ и полиномиальная функиия $\tau$ такие, что $\mathscr{B}(A, s)$ - рациональное число и

$$
\begin{aligned}
& A \in \mathfrak{C} \Longrightarrow \mathrm{P}(\mathscr{A}(A, s)=1) \geqslant \mathscr{B}(A, s)+\frac{1}{\tau(|s| n)}, \\
& A \notin \mathfrak{C} \Longrightarrow \mathrm{P}(\mathscr{A}(A, s)=1) \leqslant \mathscr{B}(A, s)-\frac{1}{\tau(|s| n)}
\end{aligned}
$$

для любой алгебраической системъ $A \subseteq \mathbb{B}^{n}$ из $\mathfrak{U}\left(n \in \mathbb{N}_{1}\right)$ и любой $Y$-порождающей последовательности s системы $A$;

2) для любой полиномиальной функиии $\tau$ существует полиномиальньй О-алгоритм $\mathscr{A}$ такой, что

$$
\mathrm{P}(\mathscr{A}(A, s)=\chi(A, \mathfrak{C})) \geqslant 1-\exp (-\tau(|s| n))
$$

для любой алгебраической системь $A \subseteq \mathbb{B}^{n}$ из $\mathfrak{U}\left(n \in \mathbb{N}_{1}\right)$ и любой $Y$-порождающей последовательности s системы $A$.

ПРЕДЛОЖЕНИЕ 3.2. Класс $\mathfrak{C}$ является (детерминированно) R-распознаваемым в классе $\mathfrak{U}$ тогда и только тогда, когда выполнено любое из следующих әквивалентных условий:

1) существуют полиномиальный (детерминированный) О-алгоритм $\mathscr{A}$, полиномиально вычислимая функиия $\varphi$, полиномиальная функиия $\tau u$ целочисленная полиномиальная функция $\rho$ такие, что $\varphi(n)-$ рациональное число при произвольном $n \in \mathbb{N}_{1}$ и для любой алгебрачческой системы $A \subseteq \mathbb{B}^{n}$ из $\mathfrak{U}\left(n \in \mathbb{N}_{1}\right)$ справедливы импликации

$$
\begin{aligned}
& A \in \mathfrak{C} \Longrightarrow \mathrm{P}(\mathscr{A}(A, \widetilde{a})=1) \geqslant \varphi(n)+\frac{1}{\tau(n)}, \\
& A \notin \mathfrak{C} \Longrightarrow \mathrm{P}(\mathscr{A}(A, \widetilde{a})=1) \leqslant \varphi(n)-\frac{1}{\tau(n)},
\end{aligned}
$$

в которьх $\widetilde{a} \in \mathrm{U} A^{\rho(n)}$

2) для любой полиномиальной функиии $\tau$ существуют полиномиальньи (детерминированный) О-алгоритм $\mathscr{A}$ и челочисленная полиномиальная функиия $\rho$ такие, что для любой алгебраической системы $A \subseteq \mathbb{B}^{n}$ из $\mathfrak{U}\left(n \in \mathbb{N}_{1}\right)$ справедливо неравенство

$$
\mathrm{P}(\mathscr{A}(A, \widetilde{a})=\chi(A, \mathfrak{C})) \geqslant 1-\exp (-\tau(n)),
$$

в котором $\widetilde{a} \in \in_{\mathrm{U}} A^{\rho(n)}$. 
Предложения 3.1 и 3.2 доказьваются с помощью стандартных рассуждений, основанных на многократном выполнении алгоритмов и применении оценки Чернова (см. доказательства вышеуказанных утверждений из [9]). Из этих предложений, в частности, вытекает, что если в определениях 3.1 и 3.2 заменить константу $2 / 3$ на любое число $q \in \mathbb{R}$, удовлетворяющее неравенствам $1 / 2<q<1$, то полученные определения будут равносильны исходным.

Лемма 3.1. Существует экспоненциальный детерминированный О-алгоритм $\mathscr{A}$ такой, что $\mathscr{A}(A, s)=A$ для любой алгебраической системы $A \subseteq \mathbb{B}^{n}$ $\left(n \in \mathbb{N}_{1}\right)$ и любой последовательности $s \in \operatorname{Gen} A$.

ДокАЗАТЕЛЬСтво. Определим экспоненциальньй детерминированньй О-алгоритм $\mathscr{A}$ следующим образом. Пусть $A \subseteq \mathbb{B}^{n}\left(n \in \mathbb{N}_{1}\right)$ - алгебраическая система и $s \in \operatorname{Gen} A$. Тогда О-алгоритм $\mathscr{A}$ при вычислении на входе $s$ строит индуктивно с помощью оракула $O_{A}$ следующие множества:

$$
\begin{aligned}
M_{0} & =E(s), \\
M_{i+1} & =M_{i} \cup\left\{\omega(a): \omega \in \Omega, a \in M_{i}^{\operatorname{ar} \omega}\right\} \quad\left(i \in \mathbb{N}_{0}\right) .
\end{aligned}
$$

Возможность построения этих множеств следует из того, что сигнатура $\Omega$ конечна. Если $M_{j+1}=M_{j}$ (что обязательно произойдет для некоторого $j \leqslant|A|-1 \leqslant$ $\left.2^{n}-1\right)$, то О-алгоритм $\mathscr{A}$ заканчивает вычисление и выдает множество $M_{j}$. Легко видеть, что $\mathscr{A}(A, s)=\bigcup_{i=0}^{\infty} M_{i}$ совпадает с подсистемой, порожденной последовательностью $s$, т.е. с $A$. Лемма доказана.

Лемма 3.2. Пусть $S$ - некоторое мнохсество такое, что $1 \leqslant|S| \leqslant N$ при некотором $N \in \mathbb{R}$. Пусть такэсе число $k \in \mathbb{N}_{1}$ удовлетворяет неравенству $k \geqslant N(\ln N+\ln 3)$. Тогда если $\widetilde{s} \in \mathrm{U} S^{k}$, mo $\mathrm{P}(E(\widetilde{s})=S) \geqslant 2 / 3$.

ДокАЗАТЕЛЬСтво. Если $N=1$, то $|S|=1$ и утверждение леммы очевидно. Считаем теперь, что $N>1$.

Пусть $\widetilde{s}=\left(\widetilde{s}_{1}, \ldots, \widetilde{s}_{k}\right)$. Тогда очевидно, что $\widetilde{s}_{1}, \ldots, \widetilde{s}_{k}-$ независимые случайные величины, распределенные равномерно на $S$. Поэтому

$$
\begin{aligned}
\mathrm{P}(E(\widetilde{s}) \neq S) & =\mathrm{P}\left(\bigcup_{s \in S} \bigcap_{i=1}^{k}\left\{\widetilde{s}_{i} \neq s\right\}\right) \leqslant|S|\left(1-\frac{1}{|S|}\right)^{k} \\
& \leqslant N\left(1-\frac{1}{N}\right)^{k}=\exp \left(k \ln \left(1-\frac{1}{N}\right)+\ln N\right) \\
& \leqslant \exp \left(-\frac{k}{N}+\ln N\right) \leqslant \exp (-\ln 3)=\frac{1}{3} .
\end{aligned}
$$

Здесь мы воспользовались легко проверяемьм неравенством $\ln q \leqslant q-1$, которое справедливо для всех $q \in \mathbb{R}_{+}$. Требуемое в лемме неравенство непосредственно вытекает из (3). Лемма доказана.

ЗАмечАниЕ 3.4. Пусть класс $\mathfrak{C}$ конечно аксиоматизируемв классе $\mathfrak{U}$. Тогда существует полиномиальный детерминированньй О-алгоритм $\mathscr{A}$ такой, что $\mathscr{A}(A, s)$ $=\chi(A, \mathfrak{C})$ для любой алгебраической системы $A \subseteq \mathbb{B}^{n}$ из $\mathfrak{U}\left(n \in \mathbb{N}_{1}\right)$ и любой последовательности $s \in A^{+}$, содержащей все элементы $A$. Этот О-алгоритм основан на переборе всех элементов входной последовательности $s$.

Из лемм 3.1 и 3.2 и замечания 3.4 вытекает 
СлЕДСТВИЕ 3.1. Пусть класс $\mathfrak{C}$ конечно аксиоматизируем в классе $\mathfrak{U}$. Тог$\partial a$

1) существует әкспоненциальный детерминированный О-алгоритм $\mathscr{A}$ такой, что $\mathscr{A}(A, s)=\chi(A, \mathfrak{C})$ для любой алгебраической системы $A \subseteq \mathbb{B}^{n}$ из $\mathfrak{U}\left(n \in \mathbb{N}_{1}\right)$ и любой последовательности $s \in \mathrm{Gen} A$;

2) существует полиномиальный детерминированньй О-алгоритм $\mathscr{A}$ такой, что если $\widetilde{a} \in_{\mathrm{U}} A^{2^{n}(n+2)}$, то $\mathrm{P}(\mathscr{A}(A, \widetilde{a})=\chi(A, \mathfrak{C})) \geqslant 2 / 3$ для любой алгебраической системы $A \subseteq \mathbb{B}^{n}$ из $\mathfrak{U}\left(n \in \mathbb{N}_{1}\right)$.

Следствие 3.1 показывает, что если класс $\mathfrak{C}$ конечно аксиоматизируем в классе $\mathfrak{U}$, то $\mathfrak{C}$ в некотором смысле детерминированно G-распознаваем в $\mathfrak{U}$ за время $\exp (\rho(|s| n))$ и детерминированно $\mathrm{R}$-распознаваем в $\mathfrak{U}$ за время $\exp (\rho(n))$, где $\rho-$ полиномиальная функция.

\section{3. Сильная $Y$-распознаваемость.}

ОПРЕДЕЛЕНИЕ 3.3. Класс $\mathfrak{C}$ называется сильно $Y$-распознаваемылм в классе $\mathfrak{U}$, если существует полиномиально вычислимая функция $\varphi$ такая, что $\varphi(m)-$ множество атомарных формул сигнатуры $\Sigma$ от переменных $\left\{t_{1}, \ldots, t_{m}\right\}$ (т.е. формул вида $u=v$ или $\pi\left(v_{1}, \ldots, v_{\operatorname{ar} \pi}\right)$, где $\pi \in \Pi$ и $\left.u, v, v_{1}, \ldots, v_{\operatorname{ar} \pi} \in \operatorname{Tm}_{m}(\Omega)\right)$ и

$$
A \in \mathfrak{C} \Longleftrightarrow \Phi(s) \text { истинно в } A \text { при всех } \Phi \in \varphi(m)
$$

для любого числа $m \in \mathbb{N}_{1}$, любой алгебраической системы $A \in \mathfrak{U}$, имеющей $Y$-порождающую последовательность длины $m$, и любой $Y$-порождающей последовательности длины $m$ системы $A$.

ЗАмечанИЕ 3.5 . Пусть $\Pi=\varnothing$ и $\mathfrak{U}$ состоит из обобщенных групп.

1) Очевидно, что класс $\mathfrak{C}$ сильно $Y$-распознаваем в классе $\mathfrak{U}$ тогда и только тогда, когда сушествует полиномиально вычислимая функция $\varphi$ такая, что $\varphi(m) \subseteq$ $\operatorname{Tm}_{m}(\Omega)$ и

$$
A \in \mathfrak{C} \Longleftrightarrow v(s)=0 \text { при всех } v \in \varphi(m)
$$

для любого числа $m \in \mathbb{N}_{1}$, любой обобщенной группы $A \in \mathfrak{U}$, имеющей $Y$-порождающую последовательность длины $m$, и любой $Y$-порождающей последовательности длины $m$ системы $A$.

2) Предположим, что $\mathfrak{C}$ является предмногообразием и $\mathfrak{U}$ замкнут относительно взятия гомоморфных образов. Тогда условие (4) выполняется для любых $m, A$ и $s$ вьшеуказанного вида в том и только том случае, когда

$$
\langle\langle v(s): v \in \varphi(m)\rangle\rangle_{A}=\mathfrak{C}(A)
$$

для любого числа $m \in \mathbb{N}_{1}$, любой обобщенной групшы $A \in \mathfrak{U}$, имеющей $Y$-порождающую последовательность длины $m$, и любой $Y$-порождающей последовательности длины $m$ системы $A$. Следовательно, при данном предположении можно заменить условие (4) на условие (5). 


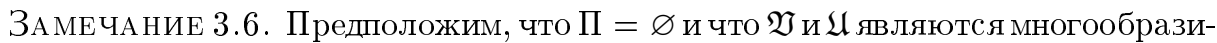
ями обобщенных групा. Обозначим через $F$ U-свободную обобщенную группу, свободно порожденную счетной системой $z_{1}, z_{2}, \ldots$ Пусть также $F_{m}$ - обобщенная группа, порожденная системой $z_{1}, \ldots, z_{m}\left(m \in \mathbb{N}_{1}\right)$.

1) Многообразие $\mathfrak{V}$ сильно G-распознаваемо в многообразии $\mathfrak{U}$, если и только если существует полиномиально вычислимая функция $\varphi$ такая, что $\varphi(m) \subseteq \operatorname{Tm}_{m}(\Omega)$ И

$$
\langle\langle v(z): v \in \varphi(m)\rangle\rangle_{F_{m}}=\mathfrak{V}\left(F_{m}\right)
$$

для любого $m \in \mathbb{N}_{1}$. Это легко вытекает из замечания 3.5 .

2 ) Пусть $\Phi$ - полугруппа всех эндоморфизмов $\varphi$ обобщенной группы $F$ таких, что $\varphi\left(z_{i}\right)=z_{\varphi^{\prime}(i)}$ при всех $i \in \mathbb{N}_{1}$, где $\varphi^{\prime}-$ некоторая строго возрастающая функция из $\mathbb{N}_{1}$ в $\mathbb{N}_{1}$ (зависяшая от $\varphi$ ). Обозначим также через $\Psi$ полугруппу всех эндоморфизмов $\psi$ обобщенной группы $F$ таких, что

$$
\psi\left(z_{i}\right) \in\{0\} \cup \bigcup_{j=1}^{\infty}\left\{-z_{j}, z_{j}\right\}
$$

для любого $i \in \mathbb{N}_{1}$. Тогда если $\mathfrak{V}(F)$ конечно порожден как $\Phi$-замкнутый (или, что эквивалентно, как $\Psi$-замкнутый) идеал, то многообразие $\mathfrak{V}$ сильно G-распознаваемо в многообразии $\mathfrak{U}$. Действительно, пусть $V$ - конечное подмножество $\operatorname{Tm}_{k}(\Omega)$ при некотором $k \in \mathbb{N}_{1}$ такое, что $\{v(z): v \in V\}$ порождает $\mathfrak{V}(F)$ как $\Phi$-замкнутый идеал. Для произвольного $m \in \mathbb{N}_{1}$ положим

$$
V_{m}=\left\{v\left(t_{i_{1}}, \ldots, t_{i_{j}}, 0, \ldots, 0\right): v \in V, 1 \leqslant i_{1}<\cdots<i_{j} \leqslant m, j \in \mathbb{I}_{k} \cup\{0\}\right\}
$$

Тогда легко видеть, что

$$
\left\langle\left\langle v(z): v \in V_{m}\right\rangle\right\rangle_{F_{m}}=\mathfrak{V}\left(F_{m}\right)
$$

Из этого равенства ввиду 1) и очевидной полиномиальной вычислимости функции $m \mapsto V_{m}\left(m \in \mathbb{N}_{1}\right)$ вытекает сильная G-распознаваемость $\mathfrak{V}$ в $\mathfrak{U}$.

\section{§4. Соотношения между различными видами распознаваемости}

4.1. Связь между (детерминированной) А-распознаваемостью и (детерминированной) R-распознаваемостью. В настоящем пункте предполагается, что класс $\mathfrak{U}$ состоит из обобщенных групп. Нам потребуется следующая лемма, проверяемая непосредственно.

ЛЕмма 4.1. Пусть $V$ и $W$ - собития в некотором вероятностном пространстве, причем $\mathrm{P}(W)>0$. Тогда $|\mathrm{P}(V)-\mathrm{P}(V \mid W)| \leqslant 1-\mathrm{P}(W)$.

ПРЕДЛОЖЕНИЕ 4.1. Пусть класс $\mathfrak{C}$ (детерминированно) А-распознаваем в классе $\mathfrak{U}$. Тогда $\mathfrak{C}$ (детерминированно) R-распознаваем в $\mathfrak{U}$. 
ДоКАЗАТЕЛЬСТВо. Выберем число $q \in \mathbb{R}_{+}$и целочисленную полиномиальную функцию $\rho$, удовлетворяющие неравенствам $q<1 / 3$ и $\rho(n) \geqslant 2 n+2+\log (1 / q)$ для всех $n \in \mathbb{N}_{1}$. Выберем также полиномиальный (детерминированный) О-алгоритм $\mathscr{A}$ такой, что

$$
\mathrm{P}(\mathscr{A}(A, s)=\chi(A, \mathfrak{C})) \geqslant \frac{2}{3}+q
$$

для любой обобщенной группы $A \subseteq \mathbb{B}^{n}$ из $\mathfrak{U}\left(n \in \mathbb{N}_{1}\right)$ и любой последовательности $s \in \operatorname{Gen} \operatorname{Add} A$. Это возможно ввиду предложения 3.1 (условие 2 )).

Пусть $A \subseteq \mathbb{B}^{n}$ - обобщенная группа из $\mathfrak{U}\left(n \in \mathbb{N}_{1}\right)$ и $\widetilde{a} \in \mathrm{U} A^{\rho(n)}$. Из результата Эрдёша и Реньи [14; теорема 1 и заключительное замечание в $\S 2]$ ввиду неравенства

$$
\rho(n) \geqslant 2 n+2+\log \frac{1}{q} \geqslant 2 \log |A|+2 \log \frac{1}{1 / 2}+\log \frac{1}{q}
$$

вытекает, что $\mathrm{P}(\widetilde{a} \in \operatorname{Gen} \operatorname{Add} A) \geqslant 1-q$. Поэтому

$$
\begin{aligned}
& |\mathrm{P}(\mathscr{A}(A, \widetilde{a})=\chi(A, \mathfrak{C}))-\mathrm{P}(\mathscr{A}(A, \widetilde{a})=\chi(A, \mathfrak{C}) \mid \widetilde{a} \in \operatorname{Gen} \operatorname{Add} A)| \\
& \quad \leqslant 1-\mathrm{P}(\widetilde{a} \in \operatorname{Gen} \operatorname{Add} A) \leqslant q
\end{aligned}
$$

согласно лемме 4.1. В то же время

$$
\mathrm{P}(\mathscr{A}(A, \widetilde{a})=\chi(A, \mathfrak{C}) \mid \widetilde{a} \in \operatorname{Gen} \operatorname{Add} A) \geqslant \frac{2}{3}+q
$$

ввиду выбора $\mathscr{A}$. Из неравенств (6) и (7) следует, что

$$
\mathrm{P}(\mathscr{A}(A, \widetilde{a})=\chi(A, \mathfrak{C})) \geqslant \frac{2}{3} .
$$

Таким образом, О-алгоритм $\mathscr{A}$ и функция $\rho$ удовлетворяют требованиям определения 3.2. Предложение доказано.

ПРЕДЛОЖЕНИЕ 4.2. Пусть класс $\mathfrak{C}$ R-распознаваем в классе $\mathfrak{U}$. Тогда $\mathfrak{C}$ А-распознаваем в $\mathfrak{U}$.

ДокАЗАТЕЛЬСТво. Фиксируем некоторое число $q \in \mathbb{R}_{+}$, удовлетворяющее неравенству $q<1 / 3$. Выберем полиномиальный О-алгоритм $\mathscr{A}$ и целочисленную полиномиальную функцию $\rho$ такие, что для любой обобщенной группы $A \subseteq \mathbb{B}^{n}$ из $\mathfrak{U}\left(n \in \mathbb{N}_{1}\right)$ справедливо неравенство

$$
\mathrm{P}(\mathscr{A}(A, \widetilde{a})=\chi(A, \mathfrak{C})) \geqslant \frac{2}{3}+q,
$$

в котором $\widetilde{a} \in \mathrm{U} A^{\rho(n)}$. Это возможно ввиду предложения 3.2 (условие 2$\left.)\right)$. Пусть также $c$ и $d$ - некоторые константы из $\mathbb{R}_{+}$такие, что $2^{-c n}+2^{-(d-1) n} \leqslant 2 q / \rho(n)$ для любого $n \in \mathbb{N}_{1}$. Из результата Бабаи [6; теорема 1.1] и его доказательства следует, что мы можем выбрать полиномиальный О-алгоритм $\mathscr{G}$, удовлетворяющий для произвольной группы $G \subseteq \mathbb{B}^{n}\left(n \in \mathbb{N}_{1}\right)$ и произвольной последовательности $f \in$ Gen $G$ следуюшим условиям:

1) $\mathscr{G}(G, f)$ всегда принадлежит $G$; 
2) существует событие $W$ (в вероятностном пространстве, на котором определены случайные биты, используемые алгоритмом $\mathscr{G}$ ) вероятности не менее $1-2^{-d n}$ такое, что

$$
\left|\mathrm{P}(\mathscr{G}(G, f)=g \mid W)-\frac{1}{|G|}\right| \leqslant \frac{2^{-c n}}{|G|}
$$

для произвольного $g \in G$.

Пусть $G \subseteq \mathbb{B}^{n}$ - группа $\left(n \in \mathbb{N}_{1}\right)$ и $f \in \operatorname{Gen} G$. Выберем событие $W$ согласно условию 2). Тогда по лемме 4.1

$$
|\mathrm{P}(\mathscr{G}(G, f)=g)-\mathrm{P}(\mathscr{G}(G, f)=g \mid W)| \leqslant 1-\mathrm{P}(W) \leqslant 2^{-d n}
$$

для любого $g \in G$. Из неравенств (8) и (9) вытекает, что

$$
\left|\mathrm{P}(\mathscr{G}(G, f)=g)-\frac{1}{|G|}\right| \leqslant \frac{2^{-c n}}{|G|}+2^{-d n} .
$$

Следовательно, если $\widetilde{g} \in_{\mathrm{U}} G$, то ввиду условия 1) справедливо неравенство

$$
\begin{aligned}
\operatorname{dist}(\mathscr{G}(G, f), \widetilde{g}) & \leqslant \frac{1}{2}\left(2^{-c n}+|G| 2^{-d n}\right) \\
& \leqslant \frac{1}{2}\left(2^{-c n}+2^{-(d-1) n}\right) \leqslant \frac{q}{\rho(n)} .
\end{aligned}
$$

Определим полиномиальный О-алгоритм $\mathscr{B}$ следующим образом. Пусть $A \subseteq \mathbb{B}^{n}$ - обобщенная группа из $\mathfrak{U}\left(n \in \mathbb{N}_{1}\right)$ и $s \in \operatorname{Gen} \operatorname{Add} A$. Тогда О-алгоритм $\mathscr{B}$ на входе $s$ выполняет $\rho(n)$ раз О-алгоритм $\mathscr{G}$ на этом входе, используя независимые друг от друга случайные биты для каждого из этих выполнений. При этом запросы О-алгоритма $\mathscr{G}$ к оракулу $O_{\text {Add } A}$ реализуются с помощью оракула $O_{A}$. Пусть при $i$-м выполнении О-алгоритм $\mathscr{G}$ выдал выходное значение $a_{i}\left(i \in \mathbb{I}_{\rho(n)}\right)$. Алгоритм $\mathscr{B}$ выполняет алгоритм $\mathscr{A}$ на входе $\left(a_{1}, \ldots, a_{\rho(n)}\right)$ и выдает полученное при этом выходное значение.

Пусть $\widetilde{r} \in \mathrm{U} \mathbb{B}^{\operatorname{NRB}(\mathscr{A}, \rho(n) n)}$ и $\widetilde{a} \in \mathrm{U} A$. Тогда из пा. 3$\left.\left.), 2\right), 5\right)$ леммы 2.1 и неравенства (10) следует, что

$$
\begin{aligned}
& \left|\mathrm{P}(\mathscr{B}(A, s)=\chi(A, \mathfrak{C}))-\mathrm{P}\left(\mathscr{A}\left(A, \widetilde{a}^{(\rho(n))}\right)=\chi(A, \mathfrak{C})\right)\right| \\
& \quad \leqslant \operatorname{dist}\left(\mathscr{A}\left(A, \mathscr{G}(\operatorname{Add} A, s)^{(\rho(n))}, \widetilde{r}\right), \mathscr{A}\left(A, \widetilde{a}^{(\rho(n))}, \widetilde{r}\right)\right) \\
& \quad \leqslant \operatorname{dist}\left(\left(\mathscr{G}(\operatorname{Add} A, s)^{(\rho(n))}, \widetilde{r}\right),\left(\widetilde{a}^{(\rho(n))}, \widetilde{r}\right)\right) \\
& \quad \leqslant \rho(n) \operatorname{dist}(\mathscr{G}(\operatorname{Add} A, s), \widetilde{a}) \leqslant q .
\end{aligned}
$$

В то же время

$$
\mathrm{P}\left(\mathscr{A}\left(A, \widetilde{a}^{(\rho(n))}\right)=\chi(A, \mathfrak{C})\right) \geqslant \frac{2}{3}+q
$$

ввиду выбора $\mathscr{A}$. Из неравенств (11) и (12) вытекает, что

$$
\mathrm{P}(\mathscr{B}(A, s)=\chi(A, \mathfrak{C})) \geqslant \frac{2}{3} .
$$

Таким образом, О-алгоритм $\mathscr{B}$ удовлетворяет требованиям определения 3.1. Предложение доказано.

Из предложений 4.1 и 4.2 непосредственно вытекает 
СЛЕДСТВИЕ 4.1. Класс $\mathfrak{C}$ А-распознаваем в классе $\mathfrak{U}$ тогда и только тогда, когда $\mathfrak{C}$ R-распознаваем в $\mathfrak{U}$.

Из определений $3.1,3.3$ и следствия 4.1 вытекает

СЛЕДСТВИЕ 4.2. Если $\mathfrak{U}$ - класс групп, то X-распознаваемость и сильная $Y$-распознаваемость $\mathfrak{C}$ в $\mathfrak{U}$ не зависят от значений соответственно $X$ и $Y$.

4.2. Сводка результатов. Связь между различньми видами распознаваемости (как полученные в п. 4.1, так и являющиеся очевидныпи замечаниями) можно выразить следуюшей диаграммой:

\begin{tabular}{|c|c|c|c|c|}
\hline $\mathrm{G}$ & $\Longrightarrow$ & $\mathrm{A}$ & $\Longleftrightarrow$ & $\mathrm{R}$ \\
\hline$\Uparrow$ & & $\Uparrow$ & & $\Uparrow$ \\
\hline DG & & $\begin{array}{c}\mathrm{DA} \\
\Uparrow\end{array}$ & $\Longrightarrow$ & DR \\
\hline $\mathrm{SG}$ & & $\mathrm{SA}$ & & \\
\hline
\end{tabular}

В этой диаграмме $X, \mathrm{D} X$ и $\mathrm{S} Y$ обозначают соответственно $X$-распознаваемость, детерминированную $X$-распознаваемость и сильную $Y$-распознаваемость. Элемент диаграммы (13) вида $Z_{1} \Longrightarrow Z_{2}\left(Z_{1} \Longleftrightarrow Z_{2}\right)$ означает, что если для классов $\mathfrak{C}$ и $\mathfrak{U}$ определена как $Z_{1}$-распознаваемость, так и $Z_{2}$-распознаваемость, то первая из них влечет вторую (соответственно первая эквивалентна второй). Отметим, что если в диаграмму (13) входят элементы $Z_{1} \Longrightarrow Z_{2}$ и $Z_{2} \Longrightarrow Z_{3}$ (или $Z_{2} \Longleftrightarrow Z_{3}$ ), то это не означает, что $Z_{1}$-распознаваемость влечет $Z_{3}$-распознаваемость в случае, когда они обе определены. Это объясняется тем, что возможны ситуации, когда определены $Z_{1}$-распознаваемость и $Z_{3}$-распознаваемость, но не определена $Z_{2}$-распознаваемость. Например, из G-распознаваемости (и даже из сильной G-распознаваемости) в общем случае не вытекает R-распознаваемость (см. п. 1.3), хотя диаграмма (13) содержит элементы $\mathrm{G} \Longrightarrow \mathrm{A}$ и $\mathrm{A} \Longleftrightarrow \mathrm{R}$.

В следующем замечании под распознаваемостью понимается либо $X$-распознаваемость, либо детерминированная $X$-распознаваемость, либо сильная $Y$-распознаваемость.

ЗАмечание 4.1. Пусть $\mathfrak{C}, \mathfrak{U}$ и $\mathfrak{V}$-классы алгебраических систем. Тогда легко видеть, что если $\mathfrak{C} \subseteq \mathfrak{U}, \mathfrak{C}$ распознаваем в $\mathfrak{U}$ и $\mathfrak{U}$ распознаваем в $\mathfrak{V}$, то $\mathfrak{C}$ распознаваем в $\mathfrak{V}$.

\section{§5. Положительные результаты}

\section{1. О сильной G-распознаваемости многообразий групп и алгебр}

Ли. В этом пункте изложение будет вестись параллельно для многообразий групп и алгебр Ли. Пусть $K$ - произвольное ассоциативное коммутативное конечно порожденное кольцо с единицей. Из теоремы Гильберта о базисе следует, что $K$ удовлетворяет условию максимальности для идеалов. Все алгебры Ли, рассматриваемые в настоящем пункте, есть линейные алгебры над кольцом $K$. Отметим, что кольца Ли можно рассматривать как алгебры Ли над кольцом целых чисел (см. также п. 2.4). Здесь мы будем обозначать через $\mathfrak{N}_{c}\left(c \in \mathbb{N}_{0}\right)$ многообразие всех нильпотентных групп (алгебр Ли) класса не более $c$, а через $\mathfrak{A}$ - многообразие всех абелевых групп (алгебр Ли). 
Пусть $A$ - произвольная группа (алгебра Ли), $S$ - некоторая система ее порождающих и $c \in \mathbb{N}_{0}$. Хорошо известно, что

$$
\mathfrak{N}_{c}(A)=A_{(c+1)}=\left\langle\left\langle\left[s_{1}^{\varepsilon_{1}}, \ldots, s_{c+1}^{\varepsilon_{c+1}}\right]: s_{i} \in S, \varepsilon_{i} \in\{-1,1\}, i \in \mathbb{I}_{c+1}\right\rangle_{A}\right.
$$

если $A-$ группа, и

$$
\mathfrak{N}_{c}(A)=\left\langle\left\langle\left(\ldots\left(\left(s_{1} s_{2}\right) s_{3}\right) \ldots\right) s_{c+1}: s_{i} \in S, i \in \mathbb{I}_{c+1}\right\rangle\right\rangle_{A}
$$

если $A$ - алгебра Ли. Отсюда ввиду замечания 3.5 вытекает

Лемма 5.1. Для любого $c \in \mathbb{N}_{0}$ многообразие $\mathfrak{N}_{c}$ сильно G-распознаваемо в классе всех групп (алгебр Ли).

Пусть $\mathfrak{U}=\mathfrak{A N}_{c} \cap \mathfrak{N}_{d} \mathfrak{A}\left(c, d \in \mathbb{N}_{0}\right)$ и $\mathfrak{V}$ - произвольное подмногообразие $\mathfrak{U}$. Тогда из результатов Вон-Ли (см. [15] для групп, а для алгебр Ли, как отмечено в [16; $§ 1]$, доказательство проводится аналогично) следует, что $\mathfrak{V}$ и $\mathfrak{U}$ удовлетворяют условиям п. 2) замечания 3.6. Поэтому имеет место

ПРЕДЛОЖЕНИЕ 5.1. Пусть $c, d \in \mathbb{N}_{0}$. Тогда любое подмногообразие $\mathfrak{A N}_{c} \cap$ $\mathfrak{N}_{d} \mathfrak{A}$ сильно G-распознаваемо в $\mathfrak{A N}_{c} \cap \mathfrak{N}_{d} \mathfrak{A}$.

Из предложения 5.1, леммы 5.1 и замечания 4.1 вытекает

СЛЕДСТВИЕ 5.1. Любое нильпотентное многообразие групп (алгебр Ли) сильно G-распознаваемо в классе всех групп (алгебр Ли).

5.2. Об $X$-распознаваемости многообразий групп. В настоящем пункте мы будем иметь дело с классами групп. Для таких классов $X$-распознаваемость и сильная $Y$-распознаваемость не зависят от значений соответственно $X$ и $Y$ ввиду следствия 4.2. Поэтому здесь мы будем говорить об $X$-распознаваемости и сильной $Y$-распознаваемости без указания значений $X$ и $Y$.

Обозначим через $\Delta$ класс всех предмногообразий групп $\mathfrak{P}$ таких, что для любой полиномиальной функции $\tau$ существует полиномиальный О-алгоритм $\mathscr{A}$, удовлетворяющий при любой групе $G \subseteq \mathbb{B}^{n}\left(n \in \mathbb{N}_{1}\right)$ и любой последовательности $s \in \operatorname{Gen} G$ следующим условиям:

1) $\mathscr{A}(G, s)$ всегда есть подмножество $\mathfrak{P}(G)$;

2) $\mathrm{P}\left(\langle\langle\mathscr{A}(G, s)\rangle\rangle_{G}=\mathfrak{P}(G)\right) \geqslant 1-\exp (-\tau(|s| n))$.

Напомним, что если $G$ - группа, то $\langle\langle S\rangle\rangle_{G}$ есть нормальное замыкание множества $S \subseteq G$ в группе $G$.

ЛЕмма 5.2. Для любой полиномиальной функиии $\tau$ существует полиномиальный О-алгоритм $\mathscr{A}$, удовлетворяющий при любой группе $G \subseteq \mathbb{B}^{n}\left(n \in \mathbb{N}_{1}\right)$ и любой последовательности $s=\left(s_{1}, \ldots, s_{2 m}\right) \in G^{+} \operatorname{ma\kappa их,~ито~}\left(s_{1}, \ldots, s_{m}\right) \in$ Gen $G$, следуюшим условиям:

1) $\mathscr{A}(G, s)$ всегда принадлежит $\left\langle\left\langle s_{m+1}, \ldots, s_{2 m}\right\rangle\right\rangle_{G}^{+}$;

2) $\mathrm{P}\left(\mathscr{A}(G, s) \in \operatorname{Gen}\left\langle\left\langle s_{m+1}, \ldots, s_{2 m}\right\rangle\right\rangle_{G}\right) \geqslant 1-\exp (-\tau(|s| n))$. 
Лемма 5.2 может быть легко выведена из результатов работы Бабаи и др. [5] и их доказательств с помощью стандартного способа повышения вероятности успеха алгоритма. В данном случае этот способ состоит в многократном вьполнении О-алгоритма и конкатенации полученных выходных значений. Здесь используется то, что если $G$ - группа и $s$ - конкатенация нескольких последовательностей из $G^{+}$, среди которых хотя бы одна принадлежит $\operatorname{Gen} G$, то $s$ также принадлежит Gen $G$.

Сформулируем нужные нам свойства класса $\Delta$.

Лемма 5.3. Пусть $\mathfrak{P}$ и $\mathfrak{Q}-$ предмногообразия групп. Тогда

1) если $\mathfrak{P} \subseteq \mathfrak{Q}, \mathfrak{P}$ сильно $Y$-распознаваемо в $\mathfrak{Q}$ и $\mathfrak{Q} \in \Delta$, то $\mathfrak{P} \in \Delta$;

2) если $\mathfrak{P}$ сильно $Y$-распознаваемо в классе всех групп, то $\mathfrak{P} \in \Delta$;

3) если $\mathfrak{P} \in \Delta$, то $\mathfrak{P} X$-распознаваемо в классе всех групп;

4) если $\mathfrak{P}$ и $\mathfrak{Q}$ - многообразия и $\mathfrak{P}, \mathfrak{Q} \in \Delta$, то $\mathfrak{P} \cap \mathfrak{Q} \in \Delta$;

5) если $\mathfrak{P}, \mathfrak{Q} \in \Delta$, mо $\mathfrak{P Q} \in \Delta$.

Пункт 1) леммы 5.3 вытекает из замечания 3.5, п. 2) - из п. 1), пп. 3) и 4) проверяются непосредственно, а п. 5) следует из леммы 5.2.

В следующем предложении через $\mathfrak{N}_{c}$, где $c \in \mathbb{N}_{0}$, обозначается многообразие всех нильпотентных групп класса не более $c$, а через $\mathfrak{A}$ - многообразие всех абелевых групп.

ПРЕДЛОЖЕНИЕ 5.2. Пусть $\mathfrak{V}_{1}, \ldots, \mathfrak{V}_{n}\left(n \in \mathbb{N}_{0}\right)-$ многообразия групn такие, что $\mathfrak{V}_{i} \subseteq \mathfrak{A N}_{c_{i}} \cap \mathfrak{N}_{d_{i}} \mathfrak{A}$ для некоторых $c_{i}, d_{i} \in \mathbb{N}_{0}$ при $i \in \mathbb{I}_{n}$. Тогда если $\mathfrak{V}=\mathfrak{V}_{1} \cdots \mathfrak{V}_{n}$, то $\mathfrak{V} \in \Delta u$, следовательно, $\mathfrak{V}$ X-распознаваемо в классе всех групп (см. п. 3) леммы 5.3).

ДокАЗАТЕЛЬСтво. Возьмем произвольное число $i \in \mathbb{I}_{n}$. Тогда из леммы 5.1 и пп. 2), 5) и 4) леммы 5.3 следует, что $\mathfrak{A N}_{c_{i}} \cap \mathfrak{N}_{d_{i}} \mathfrak{A} \in \Delta$. Поэтому $\mathfrak{V}_{i} \in \Delta$ ввиду предложения 5.1 и п. 1) леммы 5.3. Из этого согласно п. 5 ) леммы 5.3 вытекает, что $\mathfrak{V} \in \Delta$. Предложение доказано.

5.3. О сильной А-распознаваемости многообразий мультиколец. В настоящем пункте мы будем иметь дело с мультикольцами. При этом будут использоваться терминология и обозначения п. 2.8. Обозначим через $F$ свободное мультикольцо, свободно порожденное счетной системой $Z=\left\{z_{1}, z_{2}, \ldots\right\}$, а через $F_{m}-$ подмультикольцо $F$, порожденное системой $\left\{z_{1}, \ldots, z_{m}\right\}\left(m \in \mathbb{N}_{0}\right)$. Положим $F_{(n)}=(\operatorname{Add} F)_{(n)}$ для произвольного $n \in \mathbb{N}_{1}$ (т.е. $F_{(n)}-n$-й член нижнего центрального ряда группы Add $F$ ). Очевидно, что $F_{(n)}$ является идеалом в $F$. Определим эндоморфизмы $\delta_{i}, \xi_{i, j}, \eta_{i, j}\left(i, j \in \mathbb{N}_{1}\right)$ мультикольца $F$, задав их значения на $Z$ следуюшим образом:

$$
\begin{aligned}
& \delta_{i}\left(z_{i}\right)=0, \quad \xi_{i, j}\left(z_{i}\right)=z_{i}+z_{j}, \quad \eta_{i, j}\left(z_{i}\right)=z_{j} \\
& \delta_{i}\left(z_{k}\right)=z_{k}, \quad \xi_{i, j}\left(z_{k}\right)=z_{k}, \quad \eta_{i, j}\left(z_{k}\right)=z_{k} \quad \text { при } k \in \mathbb{N}_{1} \backslash\{i\} .
\end{aligned}
$$

Пусть также

$$
\lambda_{i, j}(f)=\xi_{i, j}(f)-\left(f+\eta_{i, j}(f)\right)
$$

для произвольных $f \in F$ и $i, j \in \mathbb{N}_{1}$. 
Элемент $f \in F$ называется $I$-нормальным., где $I$ - конечное подмножество $\mathbb{N}_{1}$, если

$$
\delta_{i}(f)=\left\{\begin{array}{l}
0 \text { при } i \in I, \\
f \quad \text { при } i \in \mathbb{N}_{1} \backslash I,
\end{array}\right.
$$

и нормальным, если он $J$-нормален для некоторого конечного множества $J \subseteq \mathbb{N}_{1}$. Понятие нормального элемента $F$ является аналогом понятия нормального многочлена (см. [8; гл. $1, \S 3$, п. 3.1]). Множество всех $I$-нормальных элементов из $F$ мы будем обозначать через Nor $I$.

ЗАмЕчАнИЕ 5.1. Очевидно, что нулевой элемент $I$-нормален для любого конечного множества $I \subseteq \mathbb{N}_{1}$. В то же время любой нормальный элемент $f \in$ $F \backslash\{0\}$ является $I$-нормальньм лишь для единственного множества $I=\left\{i \in \mathbb{N}_{1}\right.$ : $\left.\delta_{i}(f)=0\right\}$.

Пусть $f \in F_{m}$ для некоторого $m \in \mathbb{N}_{0}$. Хорошо известно, что для любого мультикольца $A$ и любой последовательности $a \in A^{m}$ корректно определено значение $f(a)$ (это значение равно $v(a)$, где $v$ - произвольный терм из $\operatorname{Tm}_{m}(\Omega)$ такой, что $f=v(z))$. Поэтому в этом пункте мы будем считать (мультикольцевыми) тождествами выражения вида $f=0$, где $f \in F$.

ЛЕмма 5.4. Любая (конечная) система мультикольцевых тождеств әквивалентна в классе всех мультиколец (конечной) системе тохдеств вида $f=0$, әде $f$ - нормальный әлемент $F$.

Доказательство леммы 5.4 аналогично доказательству теоремы 33.45 из [10]. Нам потребуется также следуюшая лемма, проверяемая непосредственно.

Лемма 5.5. Пусть $I$ - конечное подмнодсество $\mathbb{N}_{1}$ u $f \in \operatorname{Nor} I$. Тогда $\lambda_{i, j}(f) \in \operatorname{Nor}(I \cup\{j\})$ для любъх $i \in I u j \in \mathbb{N}_{1}$.

Лемма 5.6. Пусть $v \in \operatorname{Tm}_{\infty}(\Theta)$ и $\varphi$ - эндоморфизм $F$ такой, что $\varphi(Z)$ содержится в подгруппе $\operatorname{Add} F$, порожденной системой $Z$. Тогда

1) $v(z) \in$ Nor nvar $v$;

2) $\varphi(v(z))=\varepsilon_{1} w_{1}(z)+\cdots+\varepsilon_{n} w_{n}(z)$ для некоторьи $n \in \mathbb{N}_{0}, \varepsilon_{i} \in\{-1,1\} u$ $w_{i} \in \operatorname{Tm}_{\infty}(\Theta)$ maкux, ито $\operatorname{deg} w_{i}=\operatorname{deg} v\left(i \in \mathbb{I}_{n}\right)$.

Оба пункта леммы 5.6 легко доказываются индукцией по построению терма $v$.

Очевидно, что множество $\left\{v(z): v \in \operatorname{Tm}_{\infty}(\Theta)\right\}$ порождает Add $F$. Следовательно, $F_{(m)}$ как подгруппа Add $F$ порождается по модулю $F_{(m+1)}$ всевозможными коммутаторами вида $\left[v_{1}(z), \ldots, v_{m}(z)\right]$, где $v_{i} \in \operatorname{Tm}_{\infty}(\Theta), i \in \mathbb{I}_{m}, m \in \mathbb{N}_{1}$ (это вытекает, например, из леммы 2.2). Поэтому для любых $m \in \mathbb{N}_{1}$ и $f \in F_{(m)}$ существуют $n \in \mathbb{N}_{0}, \varepsilon_{i} \in\{-1,1\}, v_{i, j} \in \operatorname{Tm}_{\infty}(\Theta)\left(i \in \mathbb{I}_{n}, j \in \mathbb{I}_{m}\right)$ такие, что

$$
f \equiv \sum_{i=1}^{n} \varepsilon_{i}\left[v_{i, 1}(z), \ldots, v_{i, m}(z)\right] \quad\left(\bmod F_{(m+1)}\right)
$$

Представление (14) назовем несократимым, если

$$
f \not \equiv \sum_{i \in I} \varepsilon_{i}\left[v_{i, 1}(z), \ldots, v_{i, m}(z)\right] \quad\left(\bmod F_{(m+1)}\right)
$$

для любого собственного подмножества $I$ множества $\mathbb{I}_{n}$. 
ЛЕмма 5.7. Пусть представление (14) әлемента $f \in F_{(m)}\left(m \in \mathbb{N}_{1}\right)$ несократимо. Тогда если $f \in \operatorname{Nor} I$ для некоторого конечного множества $I \subseteq \mathbb{N}_{1}$, mo $\bigcup_{j=1}^{m} \operatorname{nvar} v_{i, j}=I$ для всех $i \in \mathbb{I}_{n}$.

ДокАЗАТЕЛьСтво. Для произвольного $k \in \mathbb{N}_{1}$ положим

$$
J_{k}=\left\{i \in \mathbb{I}_{n}: k \notin \bigcup_{j=1}^{m} \operatorname{nvar} v_{i, j}\right\}
$$

Тогда из п. 1) леммы 5.6 вытекает, что

$$
\delta_{k}(f) \equiv \sum_{i \in J_{k}} \varepsilon_{i}\left[v_{i, 1}(z), \ldots, v_{i, m}(z)\right] \quad\left(\bmod F_{(m+1)}\right)
$$

Пусть сначала $k \in I$. Тогда $\delta_{k}(f)=0$ и

$$
f \equiv \sum_{i \in \mathbb{I}_{n} \backslash J_{k}} \varepsilon_{i}\left[v_{i, 1}(z), \ldots, v_{i, m}(z)\right] \quad\left(\bmod F_{(m+1)}\right)
$$

ввиду (15). Следовательно, $J_{k}=\varnothing$ (так как представление (14) несократимо), т.е. $k \in \bigcup_{j=1}^{m} \operatorname{nvar} v_{i, j}$ для любого $i \in \mathbb{I}_{n}$.

Предположим теперь, что $k \in \mathbb{N}_{1} \backslash I$. Тогда $\delta_{k}(f)=f$ и $J_{k}=\mathbb{I}_{n}$ ввиду (15) и несократимости представления (14). Последнее равенство означает, что $k \notin$ $\bigcup_{j=1}^{m} \operatorname{nvar} v_{i, j}$ для всех $i \in \mathbb{I}_{n}$. Лемма доказана.

Пусть $f \in F_{(m)} \backslash F_{(m+1)}$, где $m \in \mathbb{N}_{1}$. Каждому такому $f$ мы сопоставим число

$$
\psi_{m}(f)=\min \max _{i \in \mathbb{I}_{n}} \sum_{j=1}^{m} \operatorname{deg} v_{i, j}
$$

где параметры $n$ и $v_{i, j}$ взяты из представления (14) элемента $f$, а минимум берется по всем таким представлениям (очевидно, что в каждом представлении (14) произвольного элемента из $F_{(m)} \backslash F_{(m+1)}$ мы имеем $\left.n \geqslant 1\right)$.

Лемма 5.8. Пусть $k, l, m \in \mathbb{N}_{1} u f \in F_{(m)} \backslash F_{(m+1)}$. Тогдаесли $\lambda_{k, l}(f) \notin$ $F_{(m+1)}$, mo $\psi_{m}\left(\lambda_{k, l}(f)\right) \leqslant \psi_{m}(f)$.

ДокАЗАТЕльство. Пусть (14) - некоторое представление элемента $f$, на котором достигается минимум из определения $\psi_{m}(f)$. Очевидно, что

$$
\begin{aligned}
\lambda_{k, l}(f) \equiv \sum_{i=1}^{n}\left(\varepsilon_{i}\left[\xi_{k, l}\left(v_{i, 1}(z)\right), \ldots, \xi_{k, l}\left(v_{i, m}(z)\right)\right]-\varepsilon_{i}\left[v_{i, 1}(z), \ldots, v_{i, m}(z)\right]\right. \\
\left.-\varepsilon_{i}\left[\eta_{k, l}\left(v_{i, 1}(z)\right), \ldots, \eta_{k, l}\left(v_{i, m}(z)\right)\right]\right)\left(\bmod F_{(m+1)}\right)
\end{aligned}
$$

Отсюда ввиду лемм 5.6 (п. 2)) и 2.2 вытекает сравнение

$$
\lambda_{k, l}(f) \equiv \sum_{r=1}^{s} \varkappa_{r}\left[w_{r, 1}(z), \ldots, w_{r, m}(z)\right] \quad\left(\bmod F_{(m+1)}\right)
$$


для некоторых $s \in \mathbb{N}_{1}\left(\right.$ так как $\left.\lambda_{k, l}(f) \notin F_{(m+1)}\right), \varkappa_{r} \in\{-1,1\}$ и $w_{r, j} \in \operatorname{Tm}_{\infty}(\Theta)$ таких, что $\operatorname{deg} w_{r, j}=\operatorname{deg} v_{i_{r}, j}\left(r \in \mathbb{I}_{s}, j \in \mathbb{I}_{m}\right)$. В частности,

$$
\sum_{j=1}^{m} \operatorname{deg} w_{r, j}=\sum_{j=1}^{m} \operatorname{deg} v_{i_{r}, j} \leqslant \psi_{m}(f)
$$

для любого $r \in \mathbb{I}_{s}$. Из этого непосредственно следует утверждение леммы. Лемма доказана.

Пусть теперь $N=\bigcup_{n=0}^{\infty} \operatorname{Nor} \mathbb{I}_{n}$. Каждой паре $(k, f)$, где $k \in \mathbb{N}_{0}$ и $f \in N$, сопоставим конечное множество $L_{k}(f) \subseteq F$ следующим образом. Предположим сначала, что $k \geqslant 1$ и $f \neq 0$. Пусть $n$ - единственное (ввиду замечания 5.1) число из $\mathbb{N}_{0}$ такое, что $f \in \operatorname{Nor} \mathbb{I}_{n}$. Тогда мы полагаем

$$
L_{k}(f)=\left\{\lambda_{i_{k}, n+k}\left(\ldots \lambda_{i_{2}, n+2}\left(\lambda_{i_{1}, n+1}(f)\right) \ldots\right): i_{j} \in \mathbb{I}_{n+j-1}, j \in \mathbb{I}_{k}\right\} .
$$

В частности, $L_{k}(f)=\varnothing$ при $n=0$. Если же $k=0$ или $f \neq 0$, то в качестве $L_{k}(f)$ мы выбираем $\{f\}$. Для произвольного множества $M \subseteq N$ через $L_{k}(M)$ будет обозначаться $\bigcup_{g \in M} L_{k}(g)$.

Лемма 5.9. Для любого конечного множества $M \subseteq N$ и любого числа $m \in \mathbb{N}_{0}$ существует $k \in \mathbb{N}_{0}$ такое, что $L_{k}(M) \subseteq F_{(m+1)}$ (и, следовательно, $L_{k^{\prime}}(M) \subseteq F_{(m+1)} \partial л_{\text {s }}$ всех $\left.k^{\prime} \in \mathbb{N}_{k}\right)$.

ДоКАЗАТЕЛЬство. Легко видеть, что достаточно доказать лемму лишь в случае, когда $M=\{g\}$, где $g \in F_{(m)} \backslash F_{(m+1)}$. Пусть $g \in$ Nor $\mathbb{I}_{r}$ (число $r \in \mathbb{N}_{0}$ определено однозначно ввиду замечания 5.1 , так как $g \neq 0$ ). Выберем произвольное число $k \in \mathbb{N}_{0}$, большее $\psi_{m}(g)-r$, и покажем, что $k$ удовлетворяет требованию леммы.

Пусть, напротив, сушествует $f \in L_{k}(\{g\})=L_{k}(g)$, не принадлежаший $F_{(m+1)}$. Очевидно, что $f \in F_{(m)}$. Пусть (14) - некоторое представление элемента $f$, на котором достигается минимум из определения $\psi_{m}(f)$. Переходя, если нужно, к произведению по некоторому подмножеству множества $\mathbb{I}_{n}$, мы считаем, что представление (14) несократимо. Из леммы 5.5 вытекает, что $f \in \operatorname{Nor}_{r+k}$. Фиксируем произвольное число $i \in \mathbb{I}_{n}$ (оно сушествует, так как $n \in \mathbb{N}_{1}$ ). Тогда $\bigcup_{j=1}^{m} \operatorname{nvar} v_{i, j}=\mathbb{I}_{r+k}$ по лемме 5.7. Следовательно,

$$
\begin{aligned}
\psi_{m}(g)<r+k & =\left|\bigcup_{j=1}^{m} \operatorname{nvar} v_{i, j}\right| \leqslant \sum_{j=1}^{m}\left|\operatorname{nvar} v_{i, j}\right| \\
& \leqslant \sum_{j=1}^{m} \operatorname{deg} v_{i, j} \leqslant \psi_{m}(f) \leqslant \psi_{m}(g)
\end{aligned}
$$

ввиду неравенства (1) и леммы 5.8. Таким образом, получено противоречие. Лемма доказана.

Через $\overline{\mathfrak{N}}_{c}$, где $c \in \mathbb{N}_{0}$, мы будем обозначать многообразие всех мультиколец, аддитивные группы которых нильпотентны класса не более $c$. 
ЛЕмма 5.10. Пусть $\mathfrak{V}$ - многообразие мультиколеи, конечно базируемое в классе всех мультиколеи, и с-число из $\mathbb{N}_{0}$. Тогда существует конечное мнохество $V \subseteq F$ такое, что

$$
A \in \mathfrak{V} \Longleftrightarrow f\left(s_{1}, \ldots, s_{r}\right)=0 \text { nри всех } f \in V, s_{i} \in S, i \in \mathbb{I}_{r}
$$

для любого мультикольиа $A \in \overline{\mathfrak{N}}_{c}$ и любой системь порожсдающих $S$ групnы Add $A$ (здесь $r$ - произвольное число из $\mathbb{N}_{0}$ такое, что $\left.V \subseteq F_{r}\right)$.

ДокАЗАТЕльство. Для произвольного множества $W \subseteq F$ мы будем обозначать через $W=0$ множество тождеств $\{f=0: f \in W\}$. Пусть конечное множество $M \subseteq F$ таково, что множество тождеств $M=0$ определяет многообразие $\mathfrak{V}$ в классе всех мультиколец. Применяя лемму 5.4 и заменяя переменные, мы считаем, что $M \subseteq N$. Выберем согласно лемме 5.9 число $k \in \mathbb{N}_{0}$ такое, что $L_{k}(M) \subseteq F_{(c+1)}$. Положим теперь $V=\bigcup_{j=0}^{k-1} L_{j}(M)$ и покажем, что $V$ удовлетворяет требованиям леммы.

Пусть $A$-произвольное мультикольцо из $\overline{\mathfrak{N}}_{c}$ и $S$-произвольная система порождающих группы $\operatorname{Add} A$. Если $A \in \mathfrak{V}$, то в $A$ истинно множество тождеств $M=0$ и, следовательно, множество тождеств $L_{j}(M)=0$ для любого $j \in \mathbb{N}_{0}$. В частности, выполнено условие правой части (16). Таким образом, импликация “”” эквивалентности (16) доказана.

Пусть теперь выполнено условие правой части (16). Покажем индукцией по убывающему параметру $j$, что в $A$ истинно множество тождеств $L_{j}(M)=0$ при $j=k, \ldots, 0$. При $j=k$ это верно потому, что $L_{k}(M) \subseteq F_{(c+1)}$ и $A \in \overline{\mathfrak{N}}_{c}$. Предположим теперь, что в $A$ истинно множество тождеств $L_{j+1}(M)=0$ для некоторого $j \in \mathbb{I}_{k-1} \cup\{0\}$. Возьмем произвольньй элемент $f \in L_{j}(M)$. Мы можем считать, что $f \neq 0$ (иначе истинность в $A$ тождества $f=0$ очевидна). Тогда сушествует единственное число $n \in \mathbb{N}_{0}$ такое, что $f \in \operatorname{Nor} \mathbb{I}_{n}$ (см. лемму 5.5 и замечание 5.1).

Очевидно, что $f \in F_{n}$. Если $n=0$, то истинность тождества $f=0$ в $A$ вытекает из правой части (16). Пусть теперь $n \geqslant 1$. Тогда $\lambda_{i, n+1}(f) \in L_{j+1}(M)$ для любого $i \in \mathbb{I}_{n}$ (по определению $L_{j+1}(M)$ ), следовательно, множество тождеств $\left\{\lambda_{i, n+1}(f)=0: i \in \mathbb{I}_{n}\right\}$ истинно в $A$. Но ввиду определения $\lambda_{i, n+1}$ это означает, что отображение

$$
\left(a_{1}, \ldots, a_{n}\right) \mapsto f\left(a_{1}, \ldots, a_{n}\right) \quad\left(a_{i} \in A, \quad i \in \mathbb{I}_{n}\right)
$$

дистрибутивно на $\operatorname{Add} A$. Отсюда и из условия правой части (16) вытекает истинность тождества $f=0$ в $A$. Таким образом, множество тождеств $L_{j}(M)=0$ истинно в $A$, что и требовалось.

Ввиду утверждения, доказанного в предыдущем абзаце, множество тождеств $M=0$ истинно в $A$, так как $M=L_{0}(M)$. Поэтому $A \in \mathfrak{V}$. Таким образом, импликация “£” эквивалентности (16) справедлива. Лемма доказана.

Следующая теорема является основным результатом настоящего пункта.

ТЕОрема 5.1. Предположим, что сигнатура $\Sigma=\Omega$ конечна. Пусть $\mathfrak{V}$ конечно базируемое многообразие мультиколец такое, что группа Add $A$ нильпотентна для любого $A \in \mathfrak{V}$. Тогда $\mathfrak{V}$ сильно А-распознаваемо в классе всех мультиколец. 
ДокАЗАТЕЛЬСТво. Очевидно, что $\mathfrak{V} \subseteq \overline{\mathfrak{N}}_{c}$ для некоторого $c \in \mathbb{N}_{0}$. Из леммы 5.10 следует, что $\mathfrak{V}$ сильно А-распознаваемо в $\overline{\mathfrak{N}}_{c}$. В то же время $\overline{\mathfrak{N}}_{c}$ сильно А-распознаваемо в классе всех мультиколец ввиду леммы 5.1. Отсюда и из замечания 4.1 вытекает сильная А-распознаваемость $\mathfrak{V}$ в классе всех мультиколец. Теорема доказана.

Из теоремы 5.1 нетрудно вывести

СЛЕДСТВИЕ 5.2. Пусть $\mathfrak{U}$ - класс всех линейных алгебр над произвольныцм ассочиативным коммутативным конечно порожденным кольцом с единичей. При этом сигнатура $\Sigma=\Omega$ можсет как содержать символ единичь, так и не содержать его. Тогда любое конечно базируемое подмногообразие $\mathfrak{U}$ сильно А-распознаваемо в $\mathfrak{U}$.

Следствие 5.2 охватывает также и случай колец, которые можно рассматривать как линейные алгебры над кольцом целых чисел (см. п. 2.4).

Хорошо известно (см., например, [10; теорема 34.14]), что любое многообразие нильпотентных групп конечно базируемо. Поэтому из теоремы 5.1 непосредственно вытекает вариант следствия 5.1 для групा, доказанньй ранее другим методом.

\section{§6. Некоторые вспомогательные понятия и результаты}

В настоящем параграфе предполагается, что сигнатура $\Sigma$ конечна. Фиксируем некоторый О-алгоритм $\mathscr{A}$.

6.1. Анализ вычисления О-алгоритма. Пусть $A \subseteq \mathbb{B}^{n}$ - алгебраическая система $\left(n \in \mathbb{N}_{1}\right), s \in A^{+}$и $r \in \mathbb{B} \geqslant \operatorname{NRB}(\mathscr{A},|s| n)$. Рассмотрим вычисление О-алгоритма $\mathscr{A}$ на входе $s$ с использованием оракула $O_{A}$ и последовательности битов $r$. Предположим, что при этом вычислении $\mathscr{A}$ делает $Q(\mathscr{A}, A, s, r)$ запросов к $O_{A}$. Пусть $i \in \mathbb{I}_{Q(\mathscr{A}, A, s, r)}$. Обозначим через $\mathrm{Qu}_{i}(\mathscr{A}, A, s, r) i$-й запрос О-алгоритма $\mathscr{A}$ к оракулу $O_{A}$ при рассматриваемом вычислении. Тогда $\mathrm{Qu}_{i}(\mathscr{A}, A, s, r)=\left(\sigma_{i}, a_{i}\right)$ для некоторых $\sigma_{i} \in \Sigma$ и $a_{i} \in A^{\text {ar } \sigma_{i}}$ согласно предположению о корректности всех запросов $\mathscr{A}_{\mathrm{k}} O_{A}$. Ответ оракула $O_{A}$ на $i$-й запрос О-алгоритма $\mathscr{A}$, т.е. $\sigma_{i}\left(a_{i}\right)$, мы обозначим через $h_{i}$. Положим также

$$
\begin{gathered}
\operatorname{Ans}_{i}(\mathscr{A}, A, s, r)=\left(h_{1}, \ldots, h_{i}\right), \\
\operatorname{View}_{i}(\mathscr{A}, A, s, r)=E(s) \cup\left\{h_{k}: k \in\left\{l \in \mathbb{I}_{i}: \sigma_{l} \in \Omega\right\}\right\}
\end{gathered}
$$

для $i \in \mathbb{I}_{Q(\mathscr{A}, A, s, r)} \cup\{0\}$. Последовательность $\operatorname{Ans}_{Q(\mathscr{A}, A, s, r)}(\mathscr{A}, A, s, r)$ и множество $\operatorname{View}_{Q(\mathscr{A}, A, s, r)}(\mathscr{A}, A, s, r)$ мы будем обозначать через $\operatorname{Ans}(\mathscr{A}, A, s, r)$ и $\operatorname{View}(\mathscr{A}, A, s, r)$ соответственно. Пусть $\mathrm{Que}_{i}$ и Out - не зависяшие от $A$ (детерминированные) функции такие, что

$$
\begin{gathered}
\mathrm{Que}_{i}\left(\mathscr{A}, s, \operatorname{Ans}_{i-1}(\mathscr{A}, A, s, r), r\right)=\mathrm{Qu}_{i}(\mathscr{A}, A, s, r) \\
\operatorname{Out}(\mathscr{A}, s, \operatorname{Ans}(\mathscr{A}, A, s, r), r)=\mathscr{A}(A, s, r)
\end{gathered}
$$

для всех алгебраических систем $A \subseteq \mathbb{B}^{n}\left(n \in \mathbb{N}_{1}\right)$, последовательностей $s \in A^{+}$, $r \in \mathbb{B} \geqslant \operatorname{NRB}(\mathscr{A},|s| n)$ и чисел $i \in \mathbb{I}_{Q(\mathscr{A}, A, s, r)}$.

Введенные выше обозначения используются в формулировке и доказательстве следующей леммы. 
Лемма 6.1. Пусть $i \in \mathbb{I}_{Q(\mathscr{A}, A, s, r)}$. Тогда если $\mathrm{Qu}_{i}(\mathscr{A}, A, s, r)=\left(\sigma_{i}, a_{i}\right)$, mo $E\left(a_{i}\right) \subseteq \operatorname{View}_{i-1}(\mathscr{A}, A, s, r)$.

ДокАЗАТЕЛЬСтво. Предположим противное. Выберем $k \in \mathbb{I}_{Q(\mathscr{A}, A, s, r)}$ такое, что при $i \in \mathbb{I}_{k-1}$ утверждение леммы верно, а при $i=k$-неверно. Пусть $B=\operatorname{View}_{k-1}(\mathscr{A}, A, s, r)$. Очевидно, что $E\left(a_{i}\right) \subseteq \operatorname{View}_{i-1}(\mathscr{A}, A, s, r) \subseteq B$, причем если $\sigma_{i} \in \Omega$, то $\sigma_{i}\left(a_{i}\right) \in \operatorname{View}_{i}(\mathscr{A}, A, s, r) \subseteq B$ для любого $i \in \mathbb{I}_{k-1}$ (ввиду выбора $k$ ). Поэтому мы можем превратить $B$ в алгебраическую систему такую, что $\sigma_{i}\left(a_{i}\right)$ в $B$ совпадает с $\sigma_{i}\left(a_{i}\right)$ в $A$ при всех $i \in \mathbb{I}_{k-1}$. Тогда индукция по $i$ с использованием формулы (17) показывает, что $\operatorname{Ans}_{i}(\mathscr{A}, B, s, r)=\operatorname{Ans}_{i}(\mathscr{A}, A, s, r)$ при $i \in \mathbb{I}_{k-1} \cup\{0\}$. Следовательно, $\mathrm{Qu}_{k}(\mathscr{A}, B, s, r)=\mathrm{Qu}_{k}(\mathscr{A}, A, s, r)=\left(\sigma_{k}, a_{k}\right)$ ввиду (17). В частности, последовательность $a_{k}$ должна принадлежать $B^{\text {ar } \sigma_{k}}$ согласно предположению о корректности запросов $\mathscr{A}$. Но это противоречит тому, что $E\left(a_{k}\right) \nsubseteq B$ по выбору $k$. Лемма доказана.

Ввиду леммы 6.1 можно сказать неформально, что $\operatorname{View}_{i}(\mathscr{A}, A, s, r)$ - это множество всех элементов $A$, которые "видел" О-алгоритм $\mathscr{A}$ после $i$ запросов к оракулу $O_{A}$ при рассматриваемом вычислении. Этим объясняется обозначение $\operatorname{View}_{i}(\mathscr{A}, A, s, r)$.

6.2. Последовательность PAns. В настоящем пункте мы будем предполагать, что $\Pi=\varnothing$. Таким образом, рассматриваемые алгебраические системы являются универсальными алгебрами. Положим

$$
\begin{aligned}
R(\mathscr{A})= & \{(s, \operatorname{Ans}(\mathscr{A}, A, s, r), r): A-\text { универсальная алгебра, } \\
& \left.A \subseteq \mathbb{B}^{n}, n \in \mathbb{N}_{1}, s \in A^{+}, r \in \mathbb{B}^{\geqslant} \geqslant \operatorname{NRB}(\mathscr{A},|s| n)\right\} .
\end{aligned}
$$

Пусть $(s, h, r) \in R(\mathscr{A})$, где $s=\left(s_{1}, \ldots, s_{m}\right)$ и $h=\left(h_{1}, \ldots, h_{k}\right)$. Пусть также $B-$ универсальная алгебра и $b=\left(b_{1}, \ldots, b_{m}\right) \in B^{m}$. Определим элементы $c_{i} \in B$ при $i \in \mathbb{I}_{k}$ индукцией по $i$ следующим образом. Предположим, что $c_{1}, \ldots, c_{i-1}$ уже определены. Для удобства введем следующие обозначения:

$$
f_{l}= \begin{cases}s_{l} & \text { при } l \in \mathbb{I}_{m}, \\ h_{l-m} & \text { при } l \in \mathbb{I}_{m+i-1} \backslash \mathbb{I}_{m}\end{cases}
$$

и

$$
g_{l}= \begin{cases}b_{l} & \text { при } l \in \mathbb{I}_{m}, \\ c_{l-m} & \text { при } l \in \mathbb{I}_{m+i-1} \backslash \mathbb{I}_{m} .\end{cases}
$$

Из формулы (17) и леммы 6.1 следует, что если

$$
\left(\omega_{i},\left(a_{i, 1}, \ldots, a_{i, \operatorname{ar} \omega_{i}}\right)\right)=\operatorname{Que}_{i}\left(\mathscr{A}, s,\left(h_{1}, \ldots, h_{i-1}\right), r\right),
$$

то $a_{i, j} \in\left\{f_{1}, \ldots, f_{m+i-1}\right\}$ для любого $j \in \mathbb{I}_{\text {ar } \omega_{i}}$. Для каждого такого $j$ положим $l_{i, j}=\min \left\{l \in \mathbb{I}_{m+i-1}: a_{i, j}=f_{l}\right\}$. В качестве $c_{i}$ мы выбираем $\omega_{i}\left(g_{l_{i, 1}}, \ldots, g_{l_{i, \text { ar } \omega_{i}}}\right)$ в $B$.

Положим

$$
\operatorname{PAns}(\mathscr{A}, s, h, r, B, b)=\left(c_{1}, \ldots, c_{k}\right)
$$

где элементы $c_{i}\left(i \in \mathbb{I}_{k}\right)$ построены выше. Следующие две леммы проверяются непосредственно. 
ЛЕмма 6.2. Пусть $A \subseteq \mathbb{B}^{n}$ - универсальная алгебра $\left(n \in \mathbb{N}_{1}\right)$ и $s \in A^{+}$. Тогда

$$
\operatorname{Ans}(\mathscr{A}, A, s, r)=h \Longleftrightarrow \operatorname{PAns}(\mathscr{A}, s, h, r, A, s)=h
$$

для всех $h$ и $r$ таких, что $(s, h, r) \in R(\mathscr{A})$.

ЛЕмма 6.3. Пусть В, $C$ - универсальные алгебры, $b \in B^{+}, \varphi-$ гомоморфизм из В в $C u(s, h, r) \in R(\mathscr{A})$, причем $|s|=|b|$. Тогда

$$
\varphi(\operatorname{PAns}(\mathscr{A}, s, h, r, B, b))=\operatorname{PAns}(\mathscr{A}, s, h, r, C, \varphi(b))
$$

Из лемм 6.2 и 6.3 вытекает

СЛЕДСТВИЕ 6.1. Пусть $A \subseteq \mathbb{B}^{n}, B$ - универсальные алгебрьи $\left(n \in \mathbb{N}_{1}\right)$, $b \in B^{+}$и

$$
\operatorname{Ans}(\mathscr{A}, A, \varphi(b), r)=h \Longleftrightarrow \varphi(\operatorname{PAns}(\mathscr{A}, \varphi(b), h, r, B, b))=h
$$

для всех $h$ и $r$ таких, что $(\varphi(b), h, r) \in R(\mathscr{A})$.

\section{§7. Примеры конечно базируемых многообразий, не являющихся G-распознаваемыми и $\mathrm{R}$-распознаваемыми в некотором классе полугрупп с нулем}

В настояшем параграфе в качестве $\mathfrak{U}$ мы выбираем класс всех конечных нильпотентных полугрупп с нулем, порожденных не более чем пятью элементами. При этом сигнатура может как содержать символ нуля, так и не содержать его. Под нильпотентностью полугруппы $S$ с нулем мы понимаем существование числа $m \in \mathbb{N}_{1}$ (зависящего от $S$ ) такого, что $s_{1} \cdots s_{m}=0$ для всех $s_{1}, \ldots, s_{m} \in S$. Фиксируем $e \in \mathbb{N}_{2}$. Если сигнатура содержит символ нуля, то в качестве $\mathfrak{V}$ мы берем многообразие, определенное в многообразии всех полугрупп с нулем тождеством $t_{1}^{e}=0$. Если же сигнатура не содержит символа нуля, то пусть многообразие $\mathfrak{V}$ определяется в многообразии всех полугрупп тождеством $t_{1}^{e} t_{2}=t_{1}^{e}$ или тождеством $t_{2} t_{1}^{e}=t_{1}^{e}$. Очевидно, что в классе всех полугрупп с нулем (символ которого есть в сигнатуре) как тождество $t_{1}^{e} t_{2}=t_{1}^{e}$, так и тождество $t_{2} t_{1}^{e}=t_{1}^{e}$ эквивалентно тождеству $t_{1}^{e}=0$. Целью настоящего параграфа является доказательство того, что $\mathfrak{V}$ не $\mathrm{G}$-распознаваемо и не R-распознаваемо в $\mathfrak{U}$.

Как и в $\S 6$, фиксируем произвольньй О-алгоритм $\mathscr{A}$. Выберем алфавит $Z=$ $\left\{z_{1}, \ldots, z_{5}\right\}$ из пяти элементов и обозначим через $Z_{0}$ алфавит $\left\{z_{1}, z_{2}, z_{3}\right\}$. Последовательности из $Z^{*}$ будут записываться без скобок и запятых (предполагается, что это не мешает однозначно восстанавливать по произвольной такой последовательности ее элементы). Мы называем элементы $Z$ буквами, а элементы $Z^{*}-c л о-$ вами (в алфавите $Z$ ). Через $u v$, где $u, v \in Z^{*}$, будет обозначаться конкатенация слов $u$ и $v$. Как обычно, слово $u \in Z^{*}$ называется подсловом слова $v \in Z^{*}$, если $v=v_{0} u v_{1}$ для некоторых $v_{0}, v_{1} \in Z^{*}$. Всякое подслово слова $v \in Z^{*}$, отличное от $v$, называется собственным подсловом $v$. Мы будем рассматривать $Z^{+}$как (свободную) полугруппу, умножением в которой является конкатенация.

Положим $K=\left\{w^{e}: w \in Z^{+}\right\}$. Введем также следующие обозначения:

1) $U$ - множество всех слов из $Z^{+}$, все подслова которых не принадлежат $K$; 
2) $V$ - множество всех слов из $Z^{+}$, некоторые собственные подслова которых принадлежат $K$;

3) $W$ - множество всех слов из $K$, все собственные подслова которых не принадлежат $K$.

Очевидно, что $Z^{+}$есть объединение попарно не пересекающихся множеств $U, V$ и $W$. Через $U_{i}$ и $W_{i}$, где $i \in \mathbb{N}_{1}$, мы будем обозначать множество всех слов длины не более $i$ из $U$ и $W$ соответственно.

Пусть $l \in \mathbb{N}_{e}$ и $w \in W_{l}$ (при $l \in \mathbb{I}_{e-1}$ множество $W_{l}$ пусто). Определим полугрупшы $A_{w}$ и $B$ как факторполугруппы Риса следующим образом:

$$
\begin{aligned}
A_{w} & =Z^{+} /\left(Z^{\geqslant l+1} \cup V \cup(W \backslash\{w\})\right), \\
B & =Z^{+} /\left(Z^{\geqslant l+1} \cup V \cup W\right) .
\end{aligned}
$$

Напомним, что факторполугруппа Риса полугрупшы $S$ по ее идеалу $I$ - это факторполугруппа $S$ по конгруэнции, классами которой являются $I$ (если он непуст) и $\{s\}$ при $s \in S \backslash I$. Корректность определения $A_{w}$ и $B$ следует из того, что $V \cup W^{\prime}$ является идеалом в полугруппе $Z^{+}$для любого множества $W^{\prime} \subseteq W$. Через $\delta_{w}, \varepsilon$ и $\psi_{w}$ мы будем обозначать естественные гомоморфизмы $Z^{+}$на $A_{w}, Z^{+}$на $B$ и $A_{w}$ на $B$ соответственно. В частности, $\psi_{w}\left(\delta_{w}(f)\right)=\varepsilon(f)$ для всех $f \in Z^{+}$.

Положим $n=\lceil(l+1) \log 5\rceil-2$. Тогда очевидно, что

$$
|B| \leqslant\left|A_{w}\right| \leqslant\left|Z^{+} / Z^{\geqslant l+1}\right|=\sum_{i=0}^{l} 5^{i}=\frac{5^{l+1}-1}{4}<\frac{5^{l+1}}{4} \leqslant 2^{n} .
$$

Выберем инъективные отображения $\xi_{w}: A_{w} \rightarrow \mathbb{B}^{n}$ и $\eta: B \rightarrow \mathbb{B}^{n}$ такие, что $\xi_{w}(a)=\eta\left(\psi_{w}(a)\right)$ при $a \in A_{w} \backslash\left\{\delta_{w}(w)\right\}$ и $\xi_{w}\left(\delta_{w}(w)\right)=d$ не зависит от $w$. Возможность такого выбора вытекает из (19) и инъективности $\psi_{w}$ на $A_{w} \backslash\left\{\delta_{w}(w)\right\}$. Нам потребуется следующее очевидное

ЗАмечаниЕ 7.1. Пусть $f \in Z^{+}$, причем $f \neq w$. Тогда очевидно, что $\delta_{w}(f) \neq$ $\delta_{w}(w)$. Поэтому

$$
\xi_{w}\left(\delta_{w}(f)\right)=\eta\left(\psi_{w}\left(\delta_{w}(f)\right)\right)=\eta(\varepsilon(f)) .
$$

Пусть $m \in \mathbb{N}_{1}$. Выберем некоторое число $k \in \mathbb{N}_{\mathrm{NRB}(\mathscr{A}, m n)}$. Для произвольных последовательностей $g \in\left(Z^{+} \backslash\{w\}\right)^{m}$ и $r \in \mathbb{B}^{k}$ положим

$$
D(g, r)=E\left(\operatorname{PAns}\left(\mathscr{A}, \eta(\varepsilon(g)), \operatorname{Ans}(\mathscr{A}, \eta(B), \eta(\varepsilon(g)), r), r, Z^{+}, g\right)\right) .
$$

Пусть также $\widetilde{w} \in \mathrm{U} W_{l}, \widetilde{r} \in \in_{\mathrm{U}} \mathbb{B}^{k}$ и $\widetilde{g}$ - какая-либо случайная величина, принимающая значения на множестве $\left(Z^{+} \backslash W_{l}\right)^{m}$.

Лемма 7.1. Пусть $g \in\left(Z^{+} \backslash\{w\}\right)^{m} u r \in \mathbb{B}^{k}$. Тогда если $w \notin D(g, r)$, mo

$$
\mathscr{A}\left(\xi_{w}\left(A_{w}\right), \xi_{w}\left(\delta_{w}(g)\right), r\right)=\mathscr{A}(\eta(B), \eta(\varepsilon(g)), r)
$$


ДОКАЗАТЕЛЬСТвО. Положим для краткости

$$
s=\eta(\varepsilon(g)) \text { и } h=\operatorname{Ans}(\mathscr{A}, \eta(B), s, r) .
$$

Тогда

$$
\eta\left(\varepsilon\left(\operatorname{PAns}\left(\mathscr{A}, s, h, r, Z^{+}, g\right)\right)\right)=h
$$

ввиду следствия 6.1.

По условию леммы $w \notin E(g)$ и $w \notin E\left(\operatorname{PAns}\left(\mathscr{A}, s, h, r, Z^{+}, g\right)\right)$. Следовательно,

$$
\begin{aligned}
\xi_{w}\left(\delta_{w}(g)\right) & =\eta(\varepsilon(g))=s \\
\xi_{w}\left(\delta_{w}\left(\operatorname{PAns}\left(\mathscr{A}, s, h, r, Z^{+}, g\right)\right)\right) & =\eta\left(\varepsilon\left(\operatorname{PAns}\left(\mathscr{A}, s, h, r, Z^{+}, g\right)\right)\right)=h
\end{aligned}
$$

ввиду замечания 7.1 и равенства (20). Из равенств $(22),(21)$ и следствия 6.1 вытекает, что

$$
\operatorname{Ans}\left(\mathscr{A}, \xi_{w}\left(A_{w}\right), \xi_{w}\left(\delta_{w}(g)\right), r\right)=h=\operatorname{Ans}(\mathscr{A}, \eta(B), \eta(\varepsilon(g)), r)
$$

Таким образом,

$$
\mathscr{A}\left(\xi_{w}\left(A_{w}\right), \xi_{w}\left(\delta_{w}(g)\right), r\right)=\operatorname{Out}(\mathscr{A}, s, h, r)=\mathscr{A}(\eta(B), \eta(\varepsilon(g)), r)
$$

ввиду равенств (18), (21) и (23). Лемма доказана.

ЛЕмма 7.2. Для произвольного $l \in \mathbb{N}_{e}$ справедливо неравенство

$$
\mathrm{P}(\widetilde{w} \in D(\widetilde{g}, \widetilde{r})) \leqslant \frac{\operatorname{NOQ}(\mathscr{A}, m n)}{\left|W_{l}\right|}
$$

ДоКАЗАТЕЛЬСТво. Пусть $M=\left\{D(g, r): g \in\left(Z^{+} \backslash W_{l}\right)^{m}, r \in \mathbb{B}^{k}\right\}$. Тогда

$$
\begin{aligned}
\mathrm{P}(\widetilde{w} \in D(\widetilde{g}, \widetilde{r})) & =\sum_{S \in M} \mathrm{P}(\widetilde{w} \in S) \mathrm{P}(D(\widetilde{g}, \widetilde{r})=S) \\
& =\sum_{S \in M} \frac{\left|S \cap W_{l}\right|}{\left|W_{l}\right|} \mathrm{P}(D(\widetilde{g}, \widetilde{r})=S) \leqslant \frac{\operatorname{NOQ}(\mathscr{A}, m n)}{\left|W_{l}\right|},
\end{aligned}
$$

так как $\left|S \cap W_{l}\right| \leqslant|S| \leqslant \operatorname{NOQ}(\mathscr{A}, m n)$ для любого $S \in M$. Лемма доказана.

Лемма 7.3. Пусть $u \in U \cap Z_{0}^{+}$. Тогда $\left(z_{4} u z_{5}\right)^{e} \in W$. 
ДоКАЗАТЕЛЬСТво. Положим для краткости $w_{1}=\left(z_{4} u z_{5}\right)^{e}$. Очевидно, что $w_{1} \in K$. Пусть $w_{0}$ - подслово $w_{1}$, принадлежашее $K$. Если ни $z_{4}$, ни $z_{5}$ не входит в $w_{0}$, то $w_{0}$ является подсловом $u$, что противоречит условию $u \in U$. Поэтому $z_{4+i}$ входит в $w_{0}$ для некоторого $i \in \mathbb{B}$. Очевидно, что число вхождений каждого элемента из $Z$ в слово $w_{0}$ кратно $e$, так как $w_{0}$ является $e$-й степенью некоторого слова из $Z^{+}$. Следовательно, $z_{4+i}$ входит в $w_{0}$ не менее $e$ раз. Это значит, что $w_{0}$ содержит все $e$ вхождений $z_{4+i}$ в $w_{1}$. Отсюда следует, что $z_{5-i}$ также входит в $w_{0}$, так как между первым и вторым вхождением $z_{4+i}$ в $w_{1}$ имеется вхождение $z_{5-i}$. Рассуждая аналогично предыдушему, мы получаем, что $w_{0}$ содержит все $e$ вхождений $z_{5-i}$ в слово $w_{1}$. В частности, $w_{0}$ содержит первый и последний элементы слова $w_{1}$ (равные соответственно $z_{4}$ и $z_{5}$ ). Следовательно, $w_{0}=w_{1}$ и $w_{1} \in W$. Лемма доказана.

Пусть $i \in \mathbb{N}_{1}$. Очевидно, что $\left|U \cap Z_{0}^{i}\right|$ не менњше числа слов длины $i$ в алфавите $Z_{0}$, которые не содержат подслов, являющихся непустыми квадратами (так как $e \geqslant 2$ ). Для последнего числа известна нижняя оценка вида $d_{0}^{i}$, где $d_{0}>1$. В качестве такого числа $d_{0}$ можно взять $2^{1 / 17}$ (см. $\left.[17]\right)$ или $((5+\sqrt{5}) / 2)^{1 / 18}$ (см. [18]). Следовательно, мы можем выбрать экспоненциальную функцию $\gamma$ такую, что $\left|U \cap Z_{0}^{i}\right| \geqslant \gamma(i)$ для всех $i \in \mathbb{N}_{1}$.

Лемма 7.4. Существует әкспоненциальная функция $\beta$ такая, что

$$
\operatorname{dist}\left(\mathscr{A}\left(\xi_{\widetilde{w}}\left(A_{\widetilde{w}}\right), \xi_{\widetilde{w}}\left(\delta_{\widetilde{w}}(\widetilde{g})\right)\right), \mathscr{A}(\eta(B), \eta(\varepsilon(\widetilde{g})))\right) \leqslant \frac{\operatorname{NOQ}(\mathscr{A}, m n)}{\beta(n)}
$$

при любом $l \in \mathbb{N}_{3 e}$.

ДоКАЗАТЕЛЬСТвО. Из лемм 7.1, 2.1 (п. 4)) и 7.2 следует, что

$$
\begin{aligned}
& \operatorname{dist}\left(\mathscr{A}\left(\xi_{\widetilde{w}}\left(A_{\widetilde{w}}\right), \xi_{\widetilde{w}}\left(\delta_{\widetilde{w}}(\widetilde{g})\right)\right), \mathscr{A}(\eta(B), \eta(\varepsilon(\widetilde{g})))\right) \\
& \leqslant \mathrm{P}(\widetilde{w} \in D(\widetilde{g}, \widetilde{r})) \leqslant \frac{\operatorname{NOQ}(\mathscr{A}, m n)}{\left|W_{l}\right|} .
\end{aligned}
$$

Кроме того, ввиду леммы $7.3 u \mapsto\left(z_{4} u z_{5}\right)^{e}$ - инъективное отображение из $U \cap Z_{0}^{i}$ в $W \cap Z^{(i+2) e}$ для произвольного $i \in \mathbb{N}_{1}$. Поэтому

$$
\left|W_{l}\right| \geqslant\left|W \cap Z^{\lfloor l / e\rfloor e}\right| \geqslant\left|U \cap Z_{0}^{\lfloor l / e\rfloor-2}\right| \geqslant \gamma\left(\left\lfloor\frac{l}{e}\right\rfloor-2\right) \geqslant \beta(n)
$$

для некоторой экспоненциальной функции $\beta$. Требуемое в лемме неравенство непосредственно вытекает из (24) и (25). Лемма доказана.

Tеорема 7.1. Предположим, ито существуют функиии $\varphi_{1}: \bigcup_{S}$ Gen $S \rightarrow \mathbb{R}$ и $\varkappa_{1}: \mathbb{N}_{1} \rightarrow \mathbb{R}_{+} \cup\{0\}$, удовлетворяющие для произвольной полугруппи $S \subseteq \mathbb{B}^{n^{\prime}}$ из $\mathfrak{U}\left(n^{\prime} \in \mathbb{N}_{1}\right)$ и произвольной последовательности $s \in \operatorname{Gen} S$ следующим условиям:

$$
\begin{aligned}
& S \in \mathfrak{V} \Longrightarrow \mathrm{P}(\mathscr{A}(S, s)=1) \geqslant \varphi_{1}(s)+\varkappa_{1}\left(|s| n^{\prime}\right), \\
& S \notin \mathfrak{V} \Longrightarrow \mathrm{P}(\mathscr{A}(S, s)=1) \leqslant \varphi_{1}(s)-\varkappa_{1}\left(|s| n^{\prime}\right) .
\end{aligned}
$$

Обгединение здесь берется по всевозможным полугруппам $S \subseteq \mathbb{B}^{n^{\prime}}$ из $\mathfrak{U}$ $\left(n^{\prime} \in \mathbb{N}_{1}\right)$. Тогда существует әкспоненциальная функиия $\alpha_{1}$ такая, ито

$$
\operatorname{NOQ}(\mathscr{A}, i) \geqslant \alpha_{1}(i) \varkappa_{1}(i)
$$

для любого числа $і$ вида $5(\lceil(l+1) \log 5\rceil-2)$, где $l \in \mathbb{N}_{3 e}$. 
ДоКАЗАТЕЛЬСТво. Положим $m=5$ и считаем, что случайная величина $\widetilde{g}$ всегда принимает значение $z=\left(z_{1}, \ldots, z_{5}\right)$. Выберем экспоненциальную функцию $\beta$ согласно лемме 7.4. Очевидно, что $A_{w}, B \in \mathfrak{U}, A_{w} \notin \mathfrak{V}, B \in \mathfrak{V}, \xi_{w}\left(\delta_{w}(z)\right)=$ $\eta(\varepsilon(z)) \in \operatorname{Gen} \xi_{w}\left(A_{w}\right) \cap \operatorname{Gen} \eta(B)$ для всех $w \in W_{l}$ (см. замечание 7.1). Поэтому из условия леммы и выбора $\beta$ следует, что

$$
\begin{aligned}
2 \varkappa_{1}(i) & \leqslant\left|\mathrm{P}\left(\mathscr{A}\left(\xi_{\widetilde{w}}\left(A_{\widetilde{w}}\right), \xi_{\widetilde{w}}\left(\delta_{\widetilde{w}}(z)\right)\right)=1\right)-\mathrm{P}(\mathscr{A}(\eta(B), \eta(\varepsilon(z)))=1)\right| \\
& \leqslant \operatorname{dist}\left(\mathscr{A}\left(\xi_{\widetilde{w}}\left(A_{\widetilde{w}}\right), \xi_{\widetilde{w}}\left(\delta_{\widetilde{w}}(z)\right)\right), \mathscr{A}(\eta(B), \eta(\varepsilon(z)))\right) \\
& \leqslant \frac{\operatorname{NOQ}(\mathscr{A}, i)}{\beta(n)} \leqslant \frac{\operatorname{NOQ}(\mathscr{A}, i)}{\beta^{\prime}(i)}
\end{aligned}
$$

для некоторой экспоненциальной функции $\beta^{\prime}$. Утверждение теоремы непосредственно вытекает из последнего неравенства. Теорема доказана.

TЕОРема 7.2. Предположим, что существуют функции $\varphi_{2}: \mathbb{N}_{1} \rightarrow \mathbb{R}$, $\varkappa_{2}: \mathbb{N}_{1} \rightarrow \mathbb{R}_{+} \cup\{0\} \quad$ и $\rho: \mathbb{N}_{1} \rightarrow \mathbb{N}_{1}$, удовлетворяющие для произвольной полугруппь $S \subseteq \mathbb{B}^{n^{\prime}}$ из $\mathfrak{U}\left(n^{\prime} \in \mathbb{N}_{1}\right)$ следующим условиям:

$$
\begin{aligned}
& S \in \mathfrak{V} \Longrightarrow \mathrm{P}(\mathscr{A}(S, \widetilde{s})=1) \geqslant \varphi_{2}\left(n^{\prime}\right)+\varkappa_{2}\left(n^{\prime}\right), \\
& S \notin \mathfrak{V} \Longrightarrow \mathrm{P}(\mathscr{A}(S, \widetilde{s})=1) \leqslant \varphi_{2}\left(n^{\prime}\right)-\varkappa_{2}\left(n^{\prime}\right),
\end{aligned}
$$

в которых $\widetilde{s} \in \in_{\mathrm{U}} S^{\rho\left(n^{\prime}\right)}$. Тогда существует әкспоненциальная функция $\alpha_{2}$ такая, что

$$
\rho(n)+\operatorname{NOQ}(\mathscr{A}, \rho(n) n) \geqslant \alpha_{2}(n) \varkappa_{2}(n)
$$

для любого числа п вида $\lceil(l+1) \log 5\rceil-2$, где $l \in \mathbb{N}_{3 e}$.

ДоказАТЕЛЬСтво. Пусть $\widetilde{f}_{0} \in_{\mathrm{U}} \eta(B) \cup\{d\}$ и $\widetilde{g}_{0} \in_{\mathrm{U}} U_{l} \cup\left\{z_{1}^{l+1}\right\}$. Тогда $\eta\left(\varepsilon\left(\widetilde{g}_{0}\right)\right) \in_{\mathrm{U}} \eta(B)$, так как $\varepsilon$ и $\eta$ инъективно отображают $U_{l} \cup\left\{z_{1}^{l+1}\right\}$ на $B$ и $B$ на $\eta(B)$ соответственно. Непосредственно проверяется, что

$$
\operatorname{dist}\left(\widetilde{f}_{0}, \eta\left(\varepsilon\left(\widetilde{g}_{0}\right)\right)\right)=\frac{1}{|\eta(B)|+1}=\frac{1}{\left|U_{l}\right|+2} \leqslant \frac{1}{\gamma(l)} \leqslant \frac{1}{\gamma^{\prime}(n)}
$$

для некоторой экспоненциальной функции $\gamma^{\prime}$ (так как $d \notin \eta(B),|\eta(B)|=$ $\left|U_{l} \cup\left\{z_{1}^{l+1}\right\}\right|=\left|U_{l}\right|+1$ и $\left.\left|U_{l}\right|+2 \geqslant\left|U_{l}\right| \geqslant\left|U \cap Z_{0}^{l}\right| \geqslant \gamma(l)\right)$.

Положим $\widetilde{f}=\widetilde{f}_{0}^{(\rho(n))}$ и $\widetilde{g}=\widetilde{g}_{0}^{(\rho(n))}$. Тогда из замечания 7.1, леммы 2.1 (пп. 3 ), $2), 5))$ и неравенства (26) следует, что

$$
\begin{aligned}
& \operatorname{dist}\left(\mathscr{A}\left(\xi_{\widetilde{w}}\left(A_{\widetilde{w}}\right), \widetilde{f}\right), \mathscr{A}\left(\xi_{\widetilde{w}}\left(A_{\widetilde{w}}\right), \xi_{\widetilde{w}}\left(\delta_{\widetilde{w}}(\widetilde{g})\right)\right)\right) \\
& \quad=\operatorname{dist}\left(\mathscr{A}\left(\xi_{\widetilde{w}}\left(A_{\widetilde{w}}\right), \widetilde{f}, \widetilde{r}\right), \mathscr{A}\left(\xi_{\widetilde{w}}\left(A_{\widetilde{w}}\right), \eta(\varepsilon(\widetilde{g})), \widetilde{r}\right)\right) \\
& \quad \leqslant \operatorname{dist}((\widetilde{w}, \widetilde{f}, \widetilde{r}),(\widetilde{w}, \eta(\varepsilon(\widetilde{g})), \widetilde{r})) \\
& \quad=\operatorname{dist}(\widetilde{f}, \eta(\varepsilon(\widetilde{g}))) \leqslant \rho(n) \operatorname{dist}\left(\widetilde{f}_{0}, \eta\left(\varepsilon\left(\widetilde{g}_{0}\right)\right)\right) \leqslant \frac{\rho(n)}{\gamma^{\prime}(n)} .
\end{aligned}
$$

Пусть $w \in W_{l}$. Из замечания 7.1 вытекает, что

$$
\xi_{w}\left(A_{w} \backslash\left\{\delta_{w}(w)\right\}\right)=\xi_{w}\left(\delta_{w}\left(Z^{+} \backslash\{w\}\right)\right)=\eta\left(\varepsilon\left(Z^{+} \backslash\{w\}\right)\right)=\eta\left(\varepsilon\left(Z^{+}\right)\right)=\eta(B)
$$


(равенство здесь понимается как совпадение множеств). Следовательно, носитель $\xi_{w}\left(A_{w}\right)$ совпадает с объединением носителя $\eta(B)$ и множества $\{d\}$. Кроме того, $A_{w}, B \in \mathfrak{U}, A_{w} \notin \mathfrak{V}$ и $B \in \mathfrak{V}$. Поэтому из условия леммы и п. 1$)$ леммы 2.1 следует, что

$$
\begin{aligned}
2 \varkappa_{2}(n) \leqslant & \left|\mathrm{P}\left(\mathscr{A}\left(\xi_{\widetilde{w}}\left(A_{\widetilde{w}}\right), \widetilde{f}\right)=1\right)-\mathrm{P}(\mathscr{A}(\eta(B), \eta(\varepsilon(\widetilde{g})))=1)\right| \\
\leqslant & \operatorname{dist}\left(\mathscr{A}\left(\xi_{\widetilde{w}}\left(A_{\widetilde{w}}\right), \widetilde{f}\right), \mathscr{A}(\eta(B), \eta(\varepsilon(\widetilde{g})))\right) \\
\leqslant & \left.\operatorname{dist}\left(\mathscr{A}_{\widetilde{w}}\left(\xi_{\widetilde{w}}\right), \widetilde{f}\right), \mathscr{A}\left(\xi_{\widetilde{w}}\left(A_{\widetilde{w}}\right), \xi_{\widetilde{w}}\left(\delta_{\widetilde{w}}(\widetilde{g})\right)\right)\right) \\
& \left.+\operatorname{dist}\left(\mathscr{A}_{\widetilde{w}}\left(\xi_{\widetilde{w}}\right), \xi_{\widetilde{w}}\left(\delta_{\widetilde{w}}(\widetilde{g})\right)\right), \mathscr{A}(\eta(B), \eta(\varepsilon(\widetilde{g})))\right) .
\end{aligned}
$$

Выберем экспоненциальную функцию $\beta$ согласно лемме 7.4. Тогда из неравенств $(28),(27)$ и выбора $\beta$ следует неравенство

$$
2 \varkappa_{2}(n) \leqslant \frac{\rho(n)}{\gamma^{\prime}(n)}+\frac{\operatorname{NOQ}(\mathscr{A}, \rho(n) n)}{\beta(n)} .
$$

Утверждение теоремы непосредственно вытекает из последнего неравенства. Теорема доказана.

Из теорем 7.1, 7.2 и неравенства (2) нетрудно вывести

СлЕДСТВИЕ 7.1. Многообразие $\mathfrak{V}$ не является ни G-распознаваемым, ни $\mathrm{R}-$ распознаваемым в классе $\mathfrak{U}$.

\section{Список литературы}

1. Babai L., Szemerédi E. On the complexity of matrix group problems. I // Proc. 25th Annual IEEE Symp. on Foundations of Computer Science. Singer Island, Florida, October 24-26, 1984, 1984. P. 229-240.

2. Shoup V. Lower bounds for discrete logarithms and related problems // Advances in Cryptology - EUROCRYPT'97. Proc. Internat. Conf. on the Theory and Application of Cryptographic Techniques. Konstanz, Germany, May 11-15, 1997. Berlin: Springer-Verlag, 1997. P. 256-266. (Lecture Notes in Comput. Sci. V. 1233.)

3. Нечаев В. И. К вопросу о сложности детерминированного алгоритма для дискретного логарифма // Матем. заметки. 1994. Т. 55. № 2. С. 91-101.

4. Boneh D., Lipton R. J. Algorithms for black-box fields and their application to cryptography // Advances in Cryptology - CRYPTO'96. Proc. 16th Annual Internat. Cryptology Conf. Santa Barbara, California, August 18-22, 1996. Berlin: Springer-Verlag, 1996. P. 283-297. (Lecture Notes in Comput. Sci. V. 1109.)

5. Babai L., Cooperman G., Finkelstein L., Luks E., Seress Á. Fast Monte Carlo algorithms for permutation groups // Proc. 23rd Annual ACM Symp. on Theory of Computing. New Orleans, Louisiana, May 6-8, 1991, 1991. P. 90-100; // J. Comput. System Sci. 1995. V. 50. № 2. P. 296-308.

6. Babai $L$. Local expansion of vertex-transitive graphs and random generation in finite groups // Proc. 23rd Annual ACM Symp. on Theory of Computing. New Orleans, Louisiana, May 6-8, 1991, 1991. P. $164-174$.

7. Impagliazzo R., Naor $M$. Efficient cryptographic schemes provably as secure as subset sum // J. Cryptology. 1996. V. 9. №4. P. 199-216.

8. Бахтурин Ю. А., Ольшанский А. Ю. Тождества // Итоги науки и техники. Соврем. пробл. матем. Фундам. напр. Т. 18. М.: ВИНИТИ, 1988. С. 117-240. 
9. Goldreich $O$. Introduction to complexity theory. Lecture notes for a two-semester course [1999] // http://www.wisdom.weizmann.ac.il/home/oded/public_html/cc.html.

10. Нейман Х. Многообразия групп. М.: Мир, 1969.

11. Скорняков Л. А. Элементы общей алгебры. М.: Наука, 1983.

12. Шеметков Л. А., Скиба А. С. Формации алгебраических систем. М.: Наука, 1989.

13. Китаев А., Шень А., Вяльй М. Классические и квантовые вычисления. М.: МЦНМО - YePo, 1999.

14. Erdös P., Rényi A. Probabilistic methods in group theory // J. Anal. Math. 1965. V. 14. P. 127-138.

15. Vaughan-Lee M.R. Abelian by nilpotent varieties // Quart. J. Math. Oxford Ser. (2). 1970. V. 21. № 82 . P. 193-202.

16. Vaughan-Lee M.R. Abelian-by-nilpotent varieties of Lie algebras // J. London Math. Soc. (2). 1975. V. 11. №3. P. 263-266.

17. Ekhad S. B., Zeilberger D. There are more than $2^{n / 17} n$-letter ternary square-free words // J. Integer Sequences (electronic). 1998. V. 1. Article 98.1.9. http://www.research.att.com/ $\sim$ njas/sequences/JIS/zeil.html.

18. Колпаков Р. М. О числе трехбуквенных бесквадратных и двухбуквенных бескубных слов // Тезисы докладов XII Международной конференции "Проблемы теоретической кибернетики". Нижний Новгород, 17-22 мая 1999 г. М.: Изд-во механико-математического факультета МГУ, 1999. С. 106.

Московский государственный

Поступила в редакцию

университет им. М.В. Ломоносова

13.11 .2000

E-mail : anokhin@mccme.ru 